ARTICLE

\title{
Defective folate metabolism causes germline epigenetic instability and distinguishes Hira as a phenotype inheritance biomarker
}

Georgina E. T. Blake1,2,4, Xiaohui Zhao (10 1,2, Hong wa Yung 1,2, Graham J. Burton (10 1,2, Anne C. Ferguson-Smith ${ }^{2,3}$, Russell S. Hamilton (1) ${ }^{2,3}$ \& Erica D. Watson (1) 1,2凶

The mechanism behind transgenerational epigenetic inheritance is unclear, particularly through the maternal grandparental line. We previously showed that disruption of folate metabolism in mice by the Mtrr hypomorphic mutation results in transgenerational epigenetic inheritance of congenital malformations. Either maternal grandparent can initiate this phenomenon, which persists for at least four wildtype generations. Here, we use genome-wide approaches to reveal genetic stability in the Mtrr model and genome-wide differential DNA methylation in the germline of Mtrr mutant maternal grandfathers. We observe that, while epigenetic reprogramming occurs, wildtype grandprogeny and great grandprogeny exhibit transcriptional changes that correlate with germline methylation defects. One region encompasses the Hira gene, which is misexpressed in embryos for at least three wildtype generations in a manner that distinguishes Hira transcript expression as a biomarker of maternal phenotypic inheritance.

\footnotetext{
${ }^{1}$ Department of Physiology, Development and Neuroscience, University of Cambridge, Cambridge, UK. ${ }^{2}$ Centre for Trophoblast Research, University of Cambridge, Cambridge, UK. ${ }^{3}$ Department of Genetics, University of Cambridge, Cambridge, UK. ${ }^{4}$ Present address: College of Medicine and Health, University of Exeter Medical School, Exeter, UK. ${ }^{\mathrm{e}_{\mathrm{e}}}$ ail: edw23@cam.ac.uk
} 
E nvironmental stressors can impact an individual's health and that of their progeny ${ }^{1-5}$. The phenotypic risk that persists for several generations in the absence of the stressor is termed transgenerational epigenetic inheritance (TEI) ${ }^{6}$. Although the mechanism is unclear, this non-conventional inheritance likely occurs independent of DNA base-sequence mutations and involves the inheritance of an epigenetic factor(s) via the germline ${ }^{6,7}$. Candidate factors in mammals include DNA methylation, histone modifications, and/or non-coding RNA $1,2,4,8-10$. How an epigenetic message resists reprogramming and is transmitted between one or even multiple generations to cause disease remains elusive. Few mammalian models of TEI exist and most focus on paternal inheritance $e^{1,2,8-11}$. We previously reported the $\mathrm{Mtrr}^{\mathrm{gt}}$ mouse line, a rare model of maternal grandparental TEI in which congenital malformations are transgenerationally inherited for at least four wildtype generations ${ }^{3}$ (see below, Supplementary Fig. 1a, d-e). While the germline is implicated, the epigenetic mechanism remains unclear.

MTRR (methionine synthase reductase) is a key enzyme required for one-carbon metabolism (i.e., folate and methionine metabolism; Supplementary Fig. 2) ${ }^{12-14}$. Folate is a well-known vitamin important for neural tube closure, yet its function in development is complex and poorly understood. One-carbon metabolism is required for thymidine synthesis ${ }^{15}$ and cellular methylation. Indeed, it transmits methyl groups for the methylation of homocysteine by methionine synthase (MTR) to form methionine and tetrahydrofolate ${ }^{16}$. Methionine acts as a precursor for $S$-adenosylmethionine (SAM), which serves as the sole methyl-donor for substrates involved in epigenetic regulation (e.g., DNA, RNA, histones) ${ }^{17-19}$. MTRR activates MTR through the reductive methylation of its vitamin $B_{12}$ cofactor $^{14}$ (Supplementary Fig. 2). Consequently, the progression of one-carbon metabolism requires MTRR to maintain genetic and epigenetic stability.

The hypomorphic Mtrrgt mutation reduces wildtype Mtrr transcript expression in mice ${ }^{3,12}$, which is sufficient to diminish MTR activity by $\sim 60 \%$ (ref. ${ }^{12}$ ). Consequently, the progression of one-carbon metabolism is inhibited by the Mtrrst mutation. Similar to mutations in the human MTRR gene ${ }^{13,20-22}$ or dietary folate deficiency in humans ${ }^{23}$, Mtrrtt/gt mice display hyperhomocysteinemia ${ }^{3,12}$ and macrocytic anaemia ${ }^{24}$ in adulthood, as well as altered DNA methylation patterns associated with gene misexpression ${ }^{3}$ and a broad range of incompletely penetrant developmental phenotypes at midgestation (e.g., growth defects and/or congenital malformations including heart, placenta, and neural tube closure defects) ${ }^{3}$. Therefore, $M$ trrgt $^{g t}$ mice are suitable for studying defective folate metabolism.

Crucially, the Mtrrst mouse line is a model of TEI that occurs via the maternal grandparental lineage ${ }^{3}$. Through highly controlled genetic pedigrees (Supplementary Fig. 1a, d-e), we demonstrated that an Mtrr+/gt heterozygous male or female mouse (i.e., the F0 generation) can initiate TEI of developmental phenotypes at embryonic day (E) 10.5 in the wildtype $\left(\mathrm{Mtrr}^{+/+}\right)$ descendants until the F4 generation ${ }^{3}$. This phenomenon occurs when all mice are wildtype for the Mtrr gene except for the initiating F0 $\mathrm{Mtrr}^{+/ g t}$ individual (Supplementary Fig. 1d-e). The phenotypes are similar in kind to those observed in Mtrrot/gt conceptuses (see above), though present at slightly lower frequencies ${ }^{3}$. Also, the phenotypes associate with locus-specific changes in DNA methylation that are linked to gene misexpression ${ }^{3}$ giving functional relevance to this epigenetic disruption.

Regardless of whether an F0 Mtrr ${ }^{+/ g t}$ male or female initiates TEI, the spectrum and frequency of developmental phenotypes in the F2-F4 wildtype generations are largely comparable between pedigrees when inherited via their daughters ${ }^{3}$. The exception is the F1 generation where phenotypic risk at E10.5 occurs only when F1 individuals are derived from $\mathrm{F} 0 \mathrm{Mtrr}+/ g t$ females (Supplementary Fig. 1d-e) ${ }^{3}$. That is, the F1 progeny of F0 $\mathrm{Mtrr}^{+/}$ $g^{t}$ males do not exhibit phenotypes at E10.5, reinforcing that these phenotypes are not transmitted through the male lineage (Supplementary Fig. 1d-e) $)^{3}$. However, F1 wildtype mice derived from F0 $\mathrm{Mtrr}^{+} / g t$ males display indicators of direct epigenetic inheritance including locus-specific epigenetic dysregulation in placentas at E10.5 associated with gene misexpression in the absence of gross phenotype ${ }^{3}$, a hematopoietic phenotype later in life ${ }^{24}$, and the ability of F1 wildtype females to perpetuate epigenetically inherited phenotypes to their offspring in a manner similar to those derived from an F0 $\mathrm{Mtrr}^{+/ g t}$ female (Supplementary fig. $1 \mathrm{~d}-\mathrm{e})^{3}$.

Since TEI in the Mtrr ${ }^{\text {gt }}$ model implicates the maternal lineage, we previously performed a blastocyst transfer experiment to show that phenotype inheritance occurred via the germline and was independent of the uterine environment. More specifically, F2 wildtype blastocysts derived from an F0 $\mathrm{Mtrr}^{+/ g t}$ maternal grandparent and F1 wildtype mother were transferred into the uteri of control females (Supplementary Fig. 1f) ${ }^{3}$. The risk for congenital malformations persisted after blastocyst transfer, indicating that phenotypic inheritance was not attributed to the uterine environment of the original mother but instead to the inheritance of an unknown epigenetic factor via the germline ${ }^{3}$. Therefore, we hypothesise that wildtype gametes from either $M t r r+/ g t$ maternal grandparent can initiate phenotypic inheritance via the F1 wildtype daughters for several generations ${ }^{3}$. However, the mechanism(s) of TEI remains unclear in the Mtrrgt model including the identity of the inherited epigenetic factor(s) and whether there are different epigenetic cues in the F0 male and female germlines that can initiate similar effects in their grandprogeny.

Here, we investigate the potential mechanism(s) of TEI in the Mtrrgt model using a genome-wide approach. First, we demonstrate that $\mathrm{Mtrr} \mathrm{rgt}_{\mathrm{gt}}$ mice are genetically stable and hence reassert focus on an epigenetic mechanism. Second, we show that germline DNA methylation is altered in F0 Mtrr ${ }^{+/ g t}$ males. F0 sperm were chosen for analysis because: (i) F0 Mtrr ${ }^{+/ g t}$ males initiate TEI of phenotypes in a similar manner to F0 $\mathrm{Mtrr}^{+/ g t}$ females, (ii) sperm are more experimental tractable than oocytes, and (iii) when assessing heritable effects, the uterine environment does not need to be controlled for in F0 Mtrr ${ }^{+/ g t}$ males. Even though differentially methylated regions (DMRs) in sperm of F0 Mtrr+/gt males are reprogrammed in somatic tissue of wildtype F1 and F2 progeny, our data shows evidence of transcriptional changes associated with germline epigenetic disruption that persists at least until the F3 generation. This proposed transcriptional memory of sperm DMRs includes misexpression of Hira, a gene important for chromatin stability ${ }^{25,26}$ and production of rRNA $^{27}$, which we propose as a biomarker and potential mediator of maternal phenotypic inheritance in the Mtrrgt model.

\section{Results}

Genetic stability in $\mathrm{Mtrr}^{\mathrm{gt}}$ mice. As one-carbon metabolism is directly linked to DNA synthesis ${ }^{15}$, we first addressed whether the $M$ trrgt allele influences genetic stability. Whole-genome sequencing (WGS) was performed on phenotypically normal C57Bl/6 J control embryos $(N=2)$ and $M t r r^{t} / g t$ embryos with congenital malformations $(N=6)$ derived from $M t^{g}{ }^{g t / g t}$ intercrosses (Supplementary Fig. 1a, c). DNA libraries were sequenced separately resulting in $\sim 30 \times$ coverage per embryo $\left(\sim 3.5 \times 10^{8}\right.$ paired-end reads/genome). The sequenced genomes were compared to the $\mathrm{C} 57 \mathrm{Bl} / 6 \mathrm{~J}$ reference genome to identify structural variants (SVs) and single-nucleotide polymorphisms (SNPs). 
The Mtrrst mutation was generated by a gene-trap (gt) insertion into intron nine of the Mtrr gene (Chr13) in the $129 \mathrm{P} 2 \mathrm{Ola} / \mathrm{Hsd}$ mouse strain before eight generations of backcrossing into the $\mathrm{C} 57 \mathrm{Bl} / 6 \mathrm{~J}$ strain $^{3}$. As a result, the majority of variants identified in Mtrrot/gt embryos were located on Chr13 in the genomic region surrounding the Mtrr locus (Supplementary Fig. 3a-b). These variants included the gene-trap and several SNPs that showed sequence similarity to the 129P2Ola/Hsd genome and likely persisted due to $M t_{r}{ }^{g t}$ genotype selection and regional crossover frequency. Variant identification in this region acted as an internal positive control of our bioinformatic method, demonstrating that it is capable of distinguishing genetic differences between experimental groups. Using these SNPs, we defined a $20 \mathrm{Mb}$ region of 129P2Ola/Hsd sequence surrounding the Mtrrgt allele (Fig. 1a). When this region was bioinformatically masked, C57Bl/6 J and Mtrrst/gt embryos contained a similar mean $( \pm \mathrm{sd})$ frequency of SNPs (C57Bl/6 J: 4,871 \pm 791 SNPs/

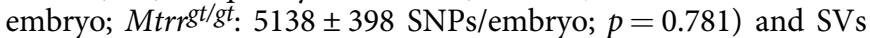
(C57Bl/6 J: 342 SVs/embryo; Mtrrst/gt: 301 SVs/embryo; $p=$ 0.6886; Fig. 1b, c) implying that the de novo mutation rate was unchanged by the Mtrrgt/gt mutation. These values were in line with expected de novo mutation rates ${ }^{28}$. Only 25 (21 SNPs and 4 SVs) variants were present in all six $M t_{r r}{ }^{g t / g t}$ embryos and absent in C57Bl/6 J embryos (Supplementary Fig. 3e, f, Supplementary Tables 1-2). When all SNPs and SVs were considered, the majority represented non-coding variants or were located in noncoding regions (Supplementary Fig. 3c, d). Moreover, genetic variation within the masked region had the minimal functional effect (beyond the gene-trap insertion) since no variant overlapped with a known enhancer, and expression of individual genes was similar among C57Bl/6 J, 129P2Ola/Hsd and Mtrrst/gt mice (Fig. 1a, d). Genomic stability was further supported by the preserved repression of transposable elements ${ }^{29,30}$ in Mtrrst/gt tissue (Fig. 1e) despite global DNA hypomethylation caused by the Mtrrgt/gt mutation ${ }^{3}$. Overall, these data support genetic integrity within the Mtrrst model, and that phenotypic inheritance was unlikely caused by an increased frequency of de novo mutation. Therefore, the focus shifted to an epigenetic mechanism.

\section{Germline DNA methylation is altered in the Mtrrgt model.} MTRR protein has a direct role in the transmission of one-carbon methyl groups for DNA methylation $3,12,14$. Therefore, germline DNA methylation was considered as a potential mediator of phenotype inheritance. As an $\mathrm{Mtrr}^{+/ g t}$ female or male can initiate TEI (Supplementary Fig. 1d-e) ${ }^{3}$ and due to the experimental tractability of male gametes, we focussed our analysis on sperm. Spermatogenesis and male fertility are normal in $\mathrm{Mtrr}^{+/+}$, $M_{t r r}+/ g t$ and $M t r r^{g t / g t}$ males $^{31}$. Mature spermatozoa were collected from caudal epididymides of $\mathrm{C} 57 \mathrm{Bl} / 6 \mathrm{~J}, \mathrm{Mtrr}^{+/+}, \mathrm{Mtrr}^{+/ g t}$ and $M t r r^{g t / g t}$ mice (Supplementary Fig. 1a-c) and sperm purity was confirmed by assessing imprinted regions of known methylation status via bisulfite pyrosequencing (Supplementary Fig. 4a). Global 5-methylcytosine (5mC) and 5-hydroxymethylcytosine $(5 \mathrm{hmC})$ levels were consistent across all Mtrr genotypes relative to $\mathrm{C} 57 \mathrm{Bl} / 6 \mathrm{~J}$ control as determined by mass spectrometry (Fig. 2a).

To analyse the genome-wide distribution of sperm DNA methylation, methylated DNA Immunoprecipitation (IP) followed by sequencing (MeDIP-seq) was performed. This approach allowed the unbiased detection of locus-specific changes in DNA methylation by identifying clusters of differentially methylated cytosines, thus reducing the potential impact of single-nucleotide variants ${ }^{4,32}$. MeDIP libraries of sperm DNA were prepared using eight males each from C57Bl/6 J, Mtrr ${ }^{+/+}, \mathrm{Mtrr}^{+/ g t}$ and Mtrrgt/gt genotypes (Supplementary Fig. 4b). Sequencing generated 179 million paired-end mappable reads on average per group $(\mathrm{C} 57 \mathrm{Bl} /$ $6 \mathrm{~J}: 164$ million reads; $M t_{r r}{ }^{+/+}$: 172 million reads; $M$ trr $^{+/ g t}$ : 203 million reads; Mtrrgt/gt: 179 million reads). Using MEDIPS package $^{33}$, each $M t r r$ genotype was independently compared to $\mathrm{C} 57 \mathrm{Bl} / 6 \mathrm{~J}$ controls. Loci of $>500 \mathrm{bp}$ with a methylation change of $>1.5$-fold and $p<0.01$ were defined as DMRs. The number of sperm DMRs identified increased with the severity of $\mathrm{Mtrr}$ genotype: 91 DMRs in $\mathrm{Mtrr}^{+/+}$males, $203 \mathrm{DMRs}$ in $\mathrm{Mtrr}^{+/ g t}$ males and 599 DMRs in Mtrrgt/gt males (Fig. 2b, c). The presence of DMRs in sperm from $\mathrm{Mtrr}^{+/+}$males indicated a parental effect of the Mtrrst allele on offspring germline methylome since $\mathrm{Mtrr}^{+/+}$males derive from $\mathrm{Mtrr}^{+}{ }_{\mathrm{gt}}$ intercrosses (Supplementary Fig. 1b). Hypo- and hypermethylated regions were identified in each Mtrr genotype when compared with $\mathrm{C} 57 \mathrm{Bl} / 6 \mathrm{~J}$ controls (Fig. 2c), consistent with earlier findings in placentas ${ }^{3}$. These data suggested that the Mtrrst allele was sufficient to dysregulate sperm DNA methylation.

To ensure the robustness and reliability of the MeDIP-seq data, we randomly selected hyper- and hypomethylated DMRs to validate using bisulfite pyrosequencing. Sperm DNA from C57Bl/ $6 \mathrm{~J}, \mathrm{Mtrr}^{+/+}, \mathrm{Mtrr}^{+/ g t}$ and $\mathrm{Mtrr} \mathrm{gt}^{\mathrm{t} / \mathrm{t}}$ males was assessed $(\mathrm{N}=8$ males/group: four sperm samples from MeDIP-seq experiment plus four independent samples). DMRs were validated in the Mtrr genotype in which they were identified (Figs. 2d, 3, Supplementary Fig. 5). The overall validation rate was $94.1 \%$ in hypomethylated DMRs and $58.3 \%$ in hypermethylated DMRs (Supplementary Table 3) and indicated a high degree of corroboration between techniques. The majority of DMRs that did not validate showed extensive methylation ( $>80 \% \mathrm{CpG}$ methylation) in $\mathrm{C} 57 \mathrm{Bl} / 6 \mathrm{~J}$ sperm and were identified as hypermethylated in the MeDIP-seq experiment (Supplementary Fig. 5). This might reflect some false positives in line with another study 4 .

For most DMRs assessed, methylation change was consistent across all $\mathrm{CpG}$ sites and the absolute change in $\mathrm{CpG}$ methylation ranged from 10 to $80 \%$ of control levels (Figs. 2d, 3, Supplementary Fig. 5). Within each genotypic group, a high degree of inter-individual consistency of methylation change was also observed. Therefore, we conclude that the Mtrrgt mutation, or parental exposure to it as in $M t r r^{+/+}$males, is sufficient to lead to distinct DNA methylation changes in sperm.

Most DMRs associate with metabolic dysregulation, not genetic effects. A proportion of the DMRs was located within the region around the gene-trap insertion site in $\mathrm{Mtrr}^{+/ g t}$ and $\mathrm{Mtr}{ }^{g t / g t}$ males (Fig. 1a, Supplementary Fig. 6b, c), consistent with $M t_{r r}^{g t / g t} \operatorname{liver}^{34}$ and suggesting that the gene-trap or underlying 129P2Ola/Hsd sequence might epigenetically dysregulate the surrounding region. However, a comparison of the MeDIP-seq and WGS data sets revealed that genetic variation did not influence DMR calling to a great extent since only a small proportion (2.8-5.5\%) of these DMRs contained one or more SNP. Eight DMRs overlapped with known enhancers (Supplementary Table 4), none of which associated with promoters ${ }^{35}$ containing a genetic variant. Outside of the Mtrr genomic region, 54 DMRs were common to $\mathrm{Mtrr}^{+/+}$, $M t r r^{+/ g t}$ and Mtrrgt/gt males (Fig. 2b, c, Supplementary Table 5) and were primarily located in distinct chromosomal clusters (Supplementary Fig. 6a-d). These data implicate epigenetic hotspots or underlying genetic effects. However, beyond a polymorphic duplication on $\mathrm{Chr} 19$ in the $\mathrm{C} 57 \mathrm{Bl} / 6 \mathrm{~J} \operatorname{strain}^{36}$ that accounted for a minor number of DMRs (2.5-15.8\% of DMRs), no DMRs overlapped with an SV or were located $<1 \mathrm{~kb}$ of an SV. Once potential genetic effects were accounted for, the majority of sperm DMRs in $\mathrm{Mtrr}^{+/+}$and $\mathrm{Mtrr}^{+/ g t}$ males (76/91 DMRs and 142/203 DMRs, respectively), and a proportion of sperm DMRs in 
a

a

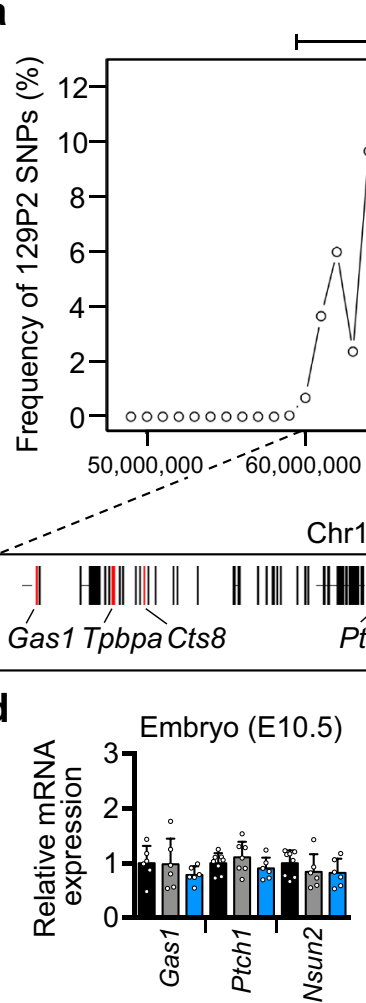

e

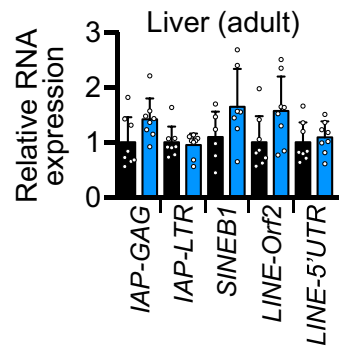

$\sim 20 \mathrm{Mb}$

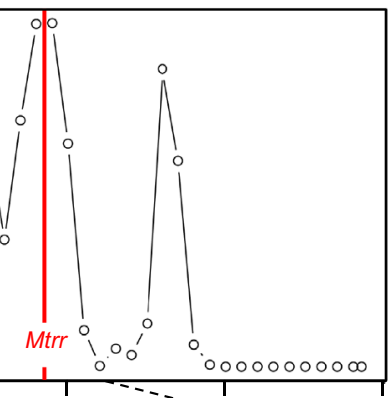

$70,000,000 \quad 80,000,000 \ldots 90,000,000 \mathrm{~kb}$

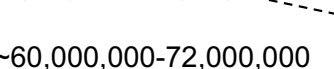

$\sim 60,000,000-72,000,000$

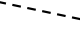

b

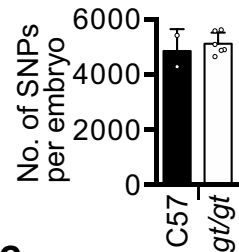

C
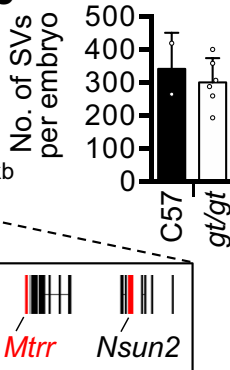

Uqcrb
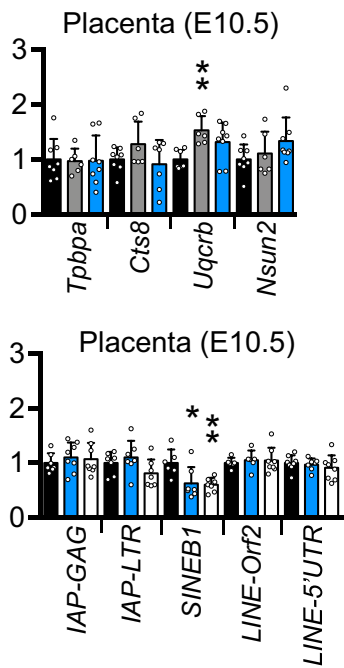

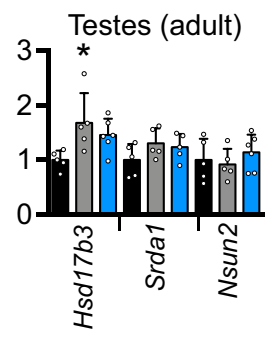

C57BI/6J

口 129P2Ola/Hsd

$\square$ Mtrrgtgt (normal)

$\square$ Mtrrgt/gt

(severely affected)

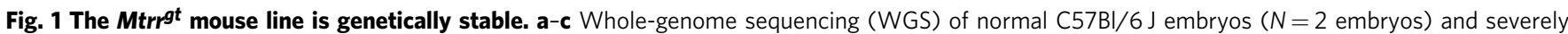
affected $M$ trrgt/gt embryos ( $N=6$ embryos) at embryonic day $(E) 10.5$ to determine the frequency of genetic variants compared to the $\mathrm{C} 57 \mathrm{Bl} / 6 \mathrm{~J}$ reference genome. a The frequency of 129P2Ola/Hsd (129P2) single-nucleotide polymorphisms (SNPs) in the region surrounding the gene-trap insertion site in the Mtrr gene (red line) on chromosome 13 (Chr13). The majority of genes within the 20 megabase $(\mathrm{Mb})$ region surrounding the Mtrr gene are shown below the graph. b, c The average number of b SNPs and c structural variants (SVs) per embryo (mean \pm standard deviation [sd]) in C57BI/6 J embryos (C57, black bars) and Mtrrgt/gt embryos ( $g t / g t$, white bars). Two-tailed unpaired $t$ test with Welch's correction. The 20 Mb region shown in a was masked when calculating the average number of genetic variants in b, c. d Graphs showing RT-qPCR analysis of selected genes (highlighted red in a) in embryos (E10.5; $N=6-8$ embryos/group), placentas (E10.5; $N=6-8$ placentas/group) and/or adult testes ( $N=5-6$ males/group) from C57Bl/6 J (black bars), $129 \mathrm{P} 2 \mathrm{Ola} /$ Hsd (grey bars) and phenotypically normal Mtrrgt/gt (blue bars) mice. One-way ANOVA with Tukey's multiple comparison test, ${ }^{\star} p=0.022,{ }^{\star \star} p=0.007$. e Graphs showing RNA expression of specific groups of transposable elements as determined by RT-qPCR in adult liver ( $N=6-8$ livers/group) and placentas (E10.5; $N=6-7$ placentas/group) from C57BI/6 J mice (black bars) and Mtrrgt/gt mice (blue bars: phenotypically normal; white bars: severely affected). Two-way ANOVA with Tukey's multiple comparison test, ${ }^{\star} p=0.011,{ }^{\star \star} p=0.005$. Data from RT-qPCR analyses in $\mathbf{d}$, e are shown as mean \pm sd and relative to $\mathrm{C} 57 \mathrm{BI} / 6 \mathrm{~J}$ tissue levels (normalised to 1). Source data are provided as a Source Data file.

Mtrrgt/gt males (174/599 DMRs) were attributed to the long-term metabolic consequences of the Mtrrst mutation.

Sperm DMR genomic distribution and potential regulatory function. DMR distribution was determined to explore the regional susceptibility of the sperm methylome to the effects of the Mtrrgt allele. First, the sperm 'background methylome' was established to resolve the expected genome-wide distribution of CpG methylation (see Methods). By comparing the regional distribution of sperm DMRs to the background methylome, we revealed that DMRs in all Mtrr genotypes were not significantly enriched in repetitive regions (Fig. 2e). However, sperm DMRs in $\mathrm{Mtrr}^{+/+}$and $\mathrm{Mtrr}{ }^{+/ g t}$ males were over-represented in introns and exons, and under-represented in intergenic regions $(p<0.0003$, Chi-squared test; Fig. 2f). This was not the case for $M$ trrot/gt males since DMRs were proportionately distributed among most genomic regions (Fig. 2f). Although the majority of sperm DMRs were located within CpG deserts, a proportion of DMRs from $\mathrm{Mtrr}^{+/ g t}$ and $\mathrm{Mtrr}^{g t / g t}$ males were enriched in CpG islands $(p<$ 0.0014, Chi-squared; Fig. 2g), which has implications for gene regulatory control. Finally, when considering only the subset of common DMRs shared by all Mtrr genotypes, a similar genomic distribution to $\mathrm{Mtrr}^{+/+}$and $\mathrm{Mtrr}{ }^{+/ g t}$ males was observed (Supplementary Fig. 6d, e).

During sperm maturation, histones are replaced by protamines $^{37}$. However, $\sim 1 \%$ of histone-containing nucleosomes 

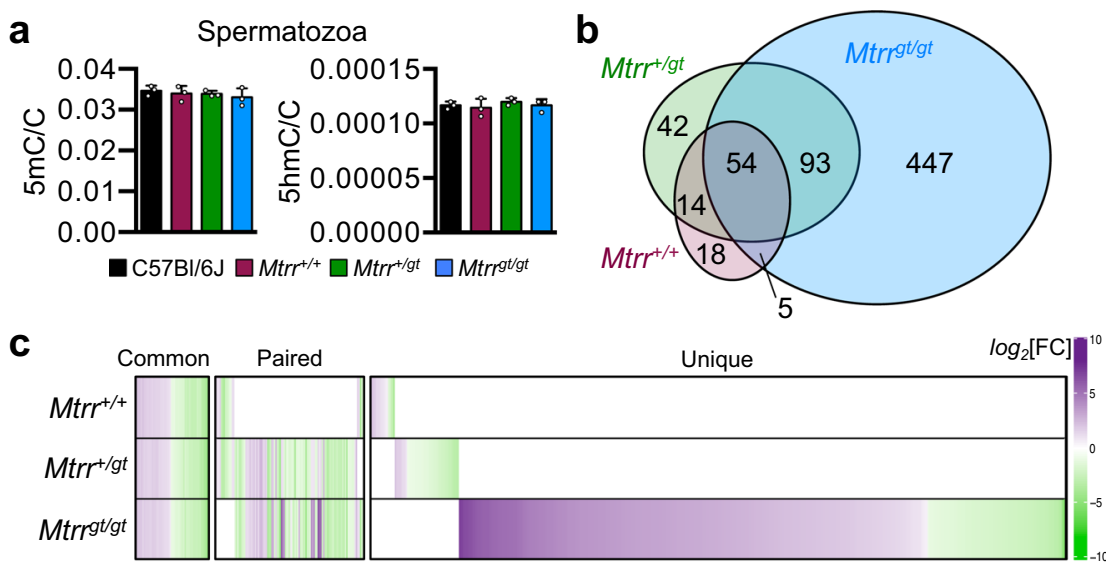

d
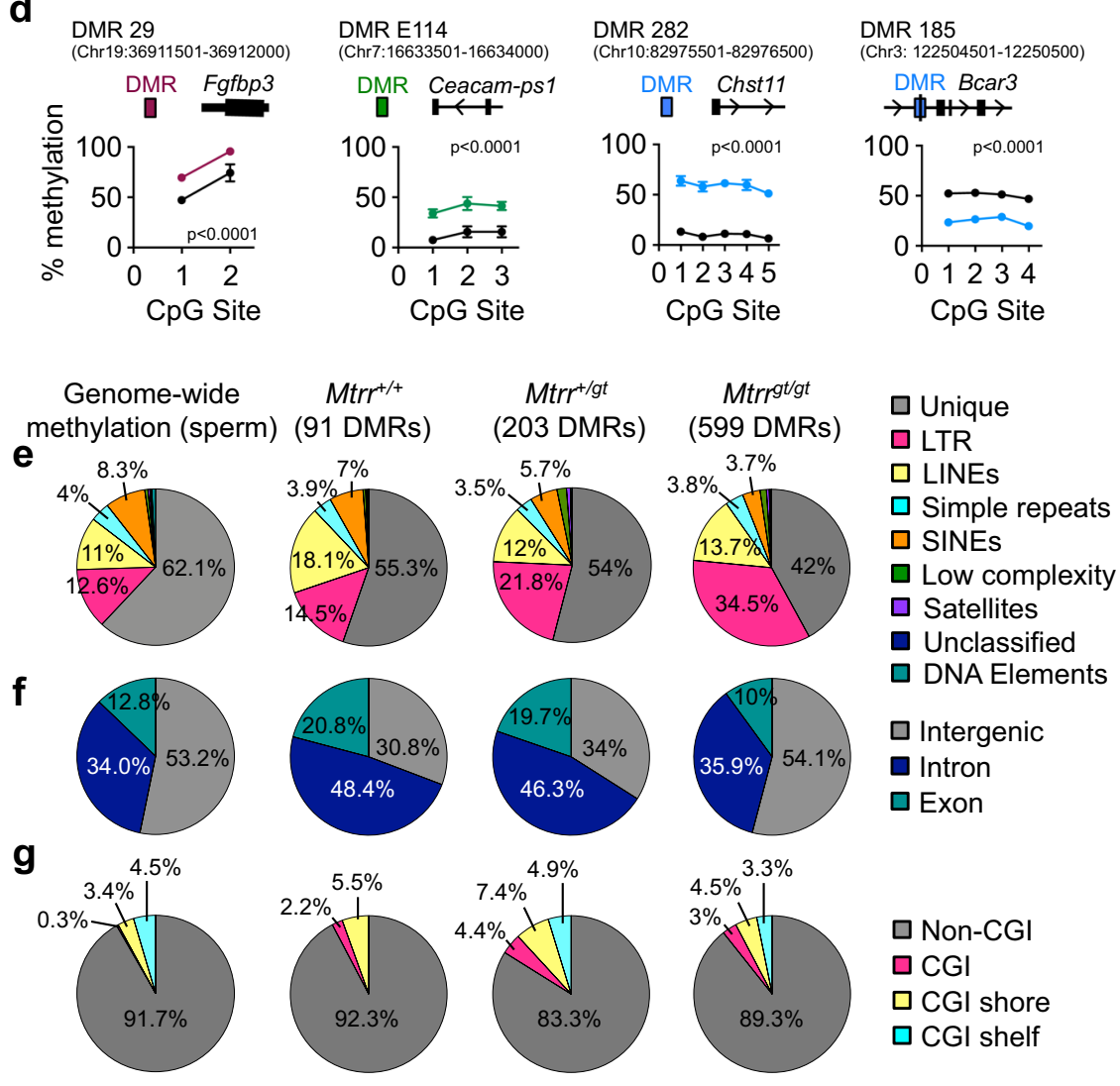

are retained ${ }^{38}$ providing scope for epigenetic inheritance ${ }^{39}$. Nucleosome retention occurs primarily at promoters of developmentally regulated genes and gene-poor repeat regions, though regional distribution and frequency differ between reports ${ }^{40,41}$. To determine whether sperm DMRs in Mtrr males were enriched in known sites of nucleosome retention, we utilised the MNaseseq data set from C57Bl/6 J spermatozoa in Erkek et al. ${ }^{41}$. First, by randomly selecting 10,000 regions of 500 bp as a proxy for DMRs, we determined that the expected frequency of DMR overlap with sites of nucleosome retention was $1.94 \%$. Crucially, we observed that $14.5-34.1 \%$ of DMRs identified in sperm from $\mathrm{Mtrr}^{+/+}$, $\mathrm{Mtrr}^{+/ g t}$ and $\mathrm{Mtrr}{ }^{g t / g t}$ males were located in nucleosome retention regions (Table 1), indicating a significant enrichment $(p<0.0001$, binomial test). Therefore, these DMRs represented candidate regions for epigenetic inheritance.

To better understand the normal epigenetic signatures within regions identified as sperm DMRs and to predict a potential gene regulatory role, mean enrichment for histone modifications and/ or chromatin accessibility in mouse spermatozoa ${ }^{42}$, epiblast and extraembryonic ectoderm at E6.5 ${ }^{43}$ was determined using published ChIP-seq and ATAC-seq data sets. All DMRs, except those surrounding the Mtrr gene-trapped site, were analyzed ( $N=379$ DMRs from all Mtrr genotypes combined) alongside 379 randomly selected regions representing the 'baseline genome' (see Methods). Compared with the sperm baseline genome, the majority of our DMRs were likely to associate with a closed chromatin state due to collective enrichment for protamine 1 (PRM1) and repressive histone mark $\mathrm{H} 3 \mathrm{~K} 9 \mathrm{me} 3$, but not active histone marks (e.g., H3K4me1, H3K27ac) or Tn5 transposase sensitive sites (THSS) ${ }^{42}$ (Supplementary Fig. 7a, b). This finding reinforces heterogeneity of DMR association with retained nucleosomes $^{41}$ or protamines (Table 1). In contrast, the DMRs were more likely in an open chromatin conformation state in epiblast and extraembryonic ectoderm at E6.5 based on collective 
Fig. 2 Characterisation of differential DNA methylation in spermatozoa from Mtrrgt mouse line. a Global 5-methylcytosine (5mC) and 5hydroxymethylcytosine $\left(5 \mathrm{hmC}\right.$ ) in spermatozoa from C57BI/6 J (black bars), wildtype (Mtrr ${ }^{+/+}$; purple bars), Mtrr + gt (green bars) and Mtrrgt/gt (blue bars) adult males ( $N=9$ males/genotype, analysed in three pools of three males/genotype) as assessed by mass spectrometry. Data are presented as the ratio of methylated cytosines per genomic cytosines $(5 \mathrm{mC} / \mathrm{C}$ or $5 \mathrm{hmC} / \mathrm{C}$; mean \pm standard deviation [sd]). One-way ANOVA. b-c Methylated DNA immunoprecipitation followed by sequencing (MeDIP-seq) of spermatozoa DNA from Mtrr ${ }^{+/+}, \mathrm{Mtrr}^{+/ g t}$ and Mtrrgt/gt males relative to C57BI/6 J control males was performed to determine differentially methylated regions (DMRs). $N=8$ males/group. $\mathbf{b}$ An intersectional analysis of DMRs in $M$ trr $+/+$ (purple), Mtrr+/gt (green), and Mtrrgt/gt (blue) relative to C57Bl/6 J controls. c A heat map plotting $\log _{2}$ FoldChange (log 2 [FC]) of CpG methylation in spermatozoa from Mtrr $+/+, M t r r^{+/ g t}$ and $M t r r^{9 t / g t}$ males compared to C57BI/6 J males $(p<0.05)$. DMRs that were common between all Mtrr genotypes, paired between two Mtrr genotypes, or unique to a single Mtrr genotype when compared to C57BI/6 J controls are shown. Relative to C57BI/6 J sperm: DMR hypermethylation shown in purple, DMR hypomethylation shown in green, normal DMR methylation shown in white. Darker colour intensity indicates greater differential methylation. $\mathbf{d}$ Examples of DMRs identified via MeDIP-seq and validated by bisulfite pyrosequencing in sperm from the male genotype in which the DMR was found. Data are shown as percentage methylation at each CpG site assessed (mean \pm sd). C57BI/6 J (black circles), Mtrr $+/+$ (purple circles), Mtrr ${ }^{+/ g t}$ (green circles) or Mtrrgt/gt (blue circles) males. $N=8$ males/group including four males from the MeDIP-seq analysis and four independent males. DMR ID and chromosomal location are shown along with a schematic of each DMR in relation to the closest gene. Two-way ANOVA with Sidak's multiple comparisons tests performed on mean methylation per CpG site per genotype group, $p<0.0001$ for all comparisons. See also Fig. 3 and Supplementary Fig. 5. e-g Relative distribution of methylated regions identified via MeDIP-seq in C57BI/6 J sperm (background methylome) and sperm DMRs from Mtrr ${ }^{+/+}, M t r r^{+/ g t}$ and $M t r r^{g t} / g t$ males among $\mathbf{e}$ unique sequences and repetitive elements, $\mathbf{f}$ coding and non-coding regions and $\mathbf{g}$ CpG islands (CGIs), shores and shelves. Statistical analyses: $\mathbf{e}, \mathbf{g}$ one-tailed Chi-squared test; $\mathbf{f}$ Two-way ANOVA with Dunnett's multiple comparison test. LTR long terminal repeats, LINEs long interspersed nuclear elements, SINEs small interspersed nuclear elements. Source data are provided as a Source Data file.

enrichment for THSS when compared with tissue-specific baseline genomes ${ }^{43}$ (Supplementary Fig. 7c). Therefore, it is possible that the genomic regions identified as DMRs in Mtrr sperm have a regulatory role during development.

Some DMRs were located in regions of reprogramming resistance. DNA methylation is largely reprogrammed during preimplantation development and in the developing germline ${ }^{44,45}$ to 'reset' the methylome between each generation. Recently, several loci were identified as 'reprogramming resistant ${ }^{46-48}$ and thus, are implicated in epigenetic inheritance. Using published methylome data sets ${ }^{46,47}$, we determined that $40.7-54.3 \%$ of sperm DMRs across all Mtrr genotypes fell within loci resistant to pre-implantation reprogramming (Table 1). Sixteen of these DMRs were common among $\mathrm{Mtrr}^{+/+}, \mathrm{Mtrr}^{+/ g t}$ and $\mathrm{Mtrr} \mathrm{s}^{\mathrm{t} / \mathrm{gt}}$ males. Fewer DMRs correlated with regions resistant to germline reprogramming (2.2-3.8\% of DMRs/Mtrr genotype; Table 1) or both pre-implantation and germline reprogramming $(2.0-2.7 \%$ of DMRs/Mtrr genotype; Table 1). Only one DMR located in a region resistant to germline reprogramming was common to all Mtrr genotypes. Furthermore, several DMRs were characterised as regions of reprogramming resistance ${ }^{46,47}$ and nucleosome retention $^{41}$ (Table 1). Overall, differential methylation of these key regions in sperm of Mtrrgt males might have important implications for epigenetic inheritance.

Sperm DMRs are reprogrammed in wildtype F1 and F2 generations. TEI in the Mtrrst model occurs via the maternal grandparental lineage ${ }^{3}$ (Supplementary Fig. 1d-e). To determine the heritability of germline DMRs, bisulfite pyrosequencing was used to validate 10 sperm DMRs from F0 Mtrr ${ }^{+/ g t}$ males in the tissue of wildtype F1 and F2 progeny (i.e., the maternal grandfather pedigree). The breeding scheme was as follows: F0 Mtrr $\mathrm{H}^{+/ g t}$ males were mated with $\mathrm{C} 57 \mathrm{Bl} / 6 \mathrm{~J}$ control females. The resulting F1 progeny was either collected at E10.5 for analysis or allowed to litter out. In the latter case, adult F1 wildtype females were mated with $\mathrm{C} 57 \mathrm{Bl} / 6 \mathrm{~J}$ control males and the $\mathrm{F} 2$ wildtype progeny was collected at E10.5 for analysis (Supplementary fig. 1d). The advantage of assessing inheritance of DNA methylation in F0 sperm rather than in F0 oocytes was that potential confounding effects of the F0 uterine environment could be avoided. Candidate DMRs were hyper- or hypomethylated, and localised to regions of reprogramming resistance and/or to intra- or intergenic regions (Supplementary Table 6). In general, all DMRs tested lost their differential methylation in wildtype F1 and F2 embryos and placentas at E10.5, and showed DNA methylation patterns similar to $\mathrm{C} 57 \mathrm{Bl} / 6 \mathrm{~J}$ tissue (Fig. 3, Supplementary Table 7). This result occurred even when wildtype F2 conceptuses displayed congenital malformations (Fig. 3). DMRs were also assessed in Mtrrgt/gt conceptuses at E10.5 to determine whether these regions were capable of differential methylation outside of the germline. In a manner similar to sperm from $\mathrm{Mtrr}^{+/ g t}$ males (Fig. 3), seven out of 10 DMRs were hypermethylated in $\mathrm{Mtr} \mathrm{rgt}^{\mathrm{g}} \mathrm{gt}$ embryos and/or placentas compared to control conceptuses (Supplementary Fig. 8a-j). In this case, it was unclear whether DNA methylation in these regions resisted epigenetic reprogramming or was erased and abnormally re-established/maintained due to intrinsic Mtrrst/gt homozygosity. Overall, altered patterns of DNA methylation in sperm of $\mathrm{Mtrr}^{+/ g t}$ males were not evident in somatic tissue of wildtype progeny and grandprogeny. This result was reminiscent of mouse models of parental exposure to environmental stressors (e.g., maternal undernutrition $^{4}$, paternal folate deficiency ${ }^{49}$, or paternal cigarette smoking ${ }^{50}$ ), which induced sperm DMRs associated with phenotypes in the direct offspring even though the DMRs were resolved in offspring somatic tissue. As a result, other epigenetic mechanisms (e.g., germline RNA content and/or histone modifications) with DNA methylation are implicated in phenotypic inheritance.

Potential transcriptional memory of sperm DMRs. A previous study in mice suggests that sperm DMRs can associate with perturbed transcription in offspring even when DNA methylation is re-established to normal levels ${ }^{4}$. To assess whether transcriptional memory associated with sperm DMRs occurred in the $M t r r^{+/ g t}$ maternal grandfather pedigree, expression of six genes located in or near sperm DMRs from F0 Mtrr ${ }^{+/ g t}$ males (Supplementary Table 6) was assessed in F1 and F2 wildtype individuals. Although all six genes displayed normal expression in F1 tissues (Fig. $4 \mathrm{a}-\mathrm{c}$ ), three of these genes including Hira (histone chaperone), Cwc27 (spliceosome-associated protein) and Tshz3 (transcription factor) were misexpressed in F2 wildtype embryos or adult livers compared to $\mathrm{C} 57 \mathrm{Bl} / 6 \mathrm{~J}$ controls (Fig. $4 \mathrm{~d}-\mathrm{f}$ ). This result might reflect transcriptional memory of the associated 


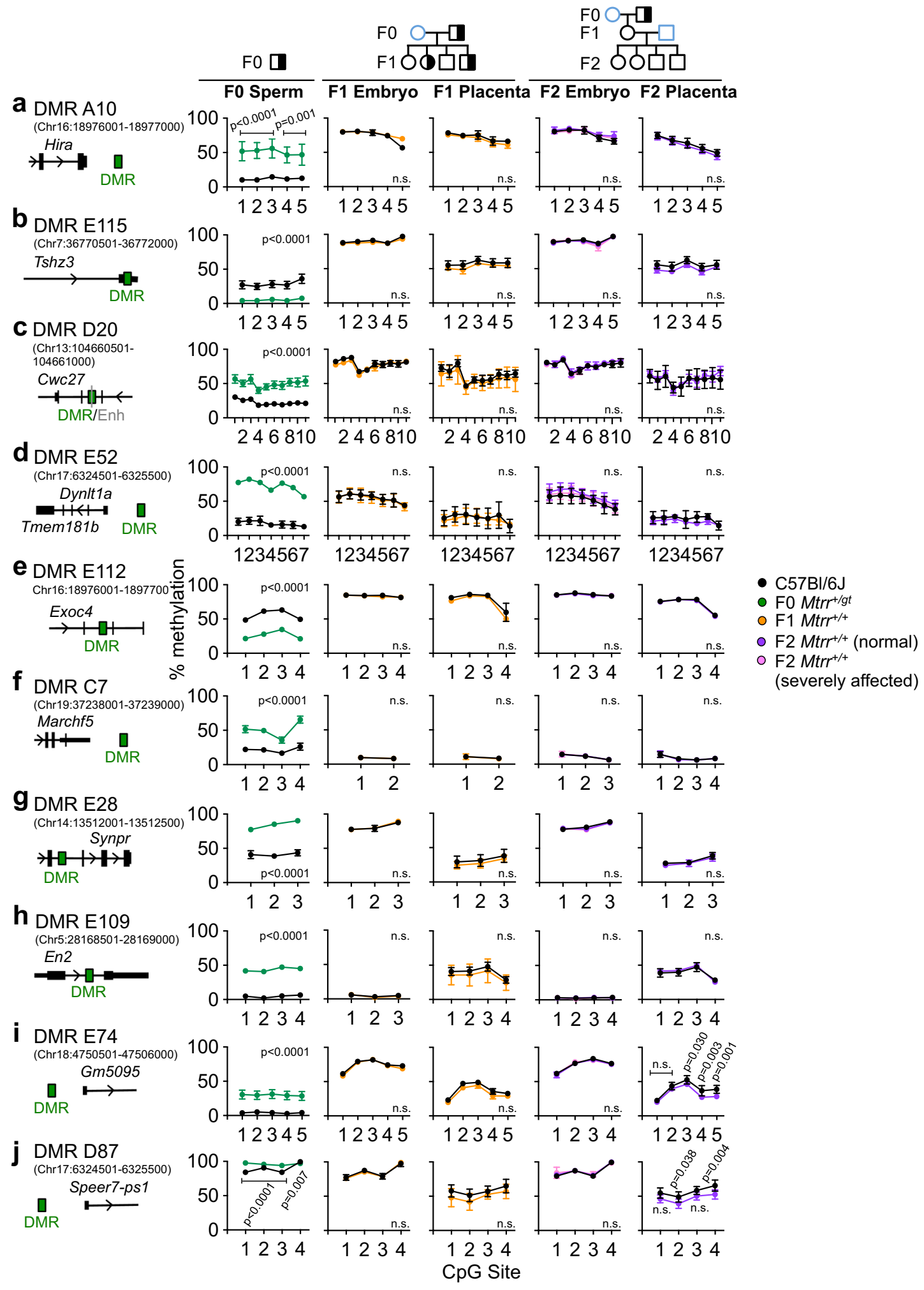

sperm DMR or wider epigenetic dysregulation in sperm of the F0 $\mathrm{Mtrr}^{+/ g t}$ males.

To further predict whether the Hira, Cwc27 and Tshz3 DMRs demarcate gene regulatory regions, their specific genetic and epigenetic characteristics beyond $\mathrm{CpG}$ methylation were considered. Genomically, the DMRs were located intragenically (Cwc27 and Tshz3 DMRs) or within $6 \mathrm{~kb}$ downstream of the gene (Hira DMR; Fig. 5a, Supplementary Figs. 9-10). Furthermore, Cwc27 DMR overlapped with a known enhancer (Supplementary
Fig. 9) while Hira and Tshz3 DMRs overlapped with CpG islands ${ }^{51}$ (Fig. 5a, Supplementary Fig. 10). Next, we assessed the three DMRs for the enrichment of specific histone modifications during normal development using published ChIP-seq data sets ${ }^{35}$ in wildtype embryonic stem cells (ESCs) and trophoblast stem cells (TSCs). Although histone marks were largely absent at all three DMRs in TSCs, enrichment for one or more methylated histone marks (e.g., H3K4me3 and/or H3K9me3) at this DMRs was apparent in ESCs (Fig. 5a, Supplementary Fig. 9-10). 
Fig. 3 Sperm DMRs are reprogrammed in somatic tissue of F1-F2 wildtype generations. CpG methylation at specific sperm differentially methylated regions (DMRs) identified in FO Mtrr ${ }^{+/ g t}$ males was assessed in the F1 and F2 wildtype embryos and placentas at E10.5. Pedigrees indicate a specific mating scheme (see also Supplementary Fig. 1a, 1d). FO, parental generation; F1 first filial generation; F2, second filial generation. a-j Schematic drawings of the sperm DMRs (green rectangles) assessed and the relationship to the closest gene and enhancer (Enh; grey line in c), if present. Graphs to the right in each panel show the average percentage of methylation at individual CpG sites for the corresponding DMR as determined by bisulfite pyrosequencing (mean \pm standard deviation [sd]). In each graph, methylation was assessed in sperm from F0 Mtrr+/gt males (green circles), phenotypically normal F1 wildtype $\left(\mathrm{Mtrr}^{+/+}\right)$embryos or placentas at E10.5 (orange circles), and phenotypically normal (purple circles) or severely affected (pink circles) F2 wildtype embryos or placentas at E10.5. C57Bl/6 J (black circles) for each tissue type are shown as controls. Specific $N$ values for each DMR and experimental group are shown in Supplementary Table 7. In brief, the following $N$ values were used (represented as a number of individuals/CpG site/ genotype): sperm: $N=3-8 ; \mathrm{F} 1$ embryos: $N=3-5 ; \mathrm{F} 1$ placentas: $N=6-8 ; \mathrm{F} 2$ embryos: $N=3-5 ; \mathrm{F} 2$ placentas: $N=3-8$. Data are shown as mean \pm sd for each CpG site. Two-way ANOVA, with Sidak's multiple comparisons test, performed on mean methylation per CpG site per genotype group. $p$ values indicated are for all CpG site comparisons within the DMR unless otherwise indicated. n.s., not significant. Pedigree legend: circle, female; square, male; blue outline, C57BI/6 J control; black outline, Mtrrgt mouse line; white filled, Mtrr ${ }^{+/+}$; half-white/half-black filled, Mtrr+/gt. Source data are provided as a Source Data file.

\begin{tabular}{|c|c|c|c|}
\hline Location of sperm DMRs & Mtrr $+/+$ & Mtrr $+/ g t$ & Mtrrgt/gt \\
\hline Regions of nucleosome retention ${ }^{41}$ & $31(34.1 \%)$ & $57(28.1 \%)$ & $87(14.5 \%)$ \\
\hline Reprogramming resistant regions in pre-implantation embryo 47 & $37(40.7 \%)$ & $96(47.3 \%)$ & $325(54.3 \%)$ \\
\hline Reprogramming resistant regions in germline 46 & $2(2.2 \%)$ & $5(2.5 \%)$ & $23(3.8 \%)$ \\
\hline Regions of nucleosome retention ${ }^{41} \&$ resistant to reprogramming in germline ${ }^{46}$ & $1(1.1 \%)$ & $2(1.0 \%)$ & $4(0.67 \%)$ \\
\hline
\end{tabular}

Altogether, DMRs identified in sperm of $\mathrm{Mtrr}^{+/ g t}$ males highlight regions that might be important for transcriptional regulation in the early conceptus by other epigenetic mechanisms. Therefore, future analyses of broader epigenetic marks at these sites are required in the Mtrrot mouse line.

HIRA as a potential biomarker of maternal phenotypic inheritance. The importance of the Hira DMR in phenotypic inheritance was further considered based on its known resistance to germline reprogramming ${ }^{46}$, and its potential function in gene regulation (Fig. 5a) and transcriptional memory (Fig. 4d). HIRA is a histone H3.3 chaperone, which lends itself well to a role in epigenetic inheritance given its broad functionality in transcriptional regulation ${ }^{26}$, maintenance of chromatin structure in the developing oocyte $\mathrm{e}^{25}$ and the male pronucleus after fertilisation ${ }^{27}$, and in rRNA transcription ${ }^{27}$. Hira ${ }^{-1-}$ mice $^{52}$ and the Mtrrst mouse line ${ }^{3}$ display similar phenotypes including growth defects, congenital malformations, and embryonic lethality by E10.5. Furthermore, $M t r r^{g t}$ genotypic severity correlated with the degree of hypermethylation in the Hira DMR in sperm (Fig. 5b, c), suggesting that the Hira DMR is responsive to alterations in folate metabolism.

The potential regulatory legacy of the Hira DMR in sperm was further explored in the $M t r r^{+/ g t}$ maternal grandfather pedigree. The Hira DMR, which was $6 \mathrm{~kb}$ downstream of the Hira gene and overlaps with a CpG island and CTCF binding site in ESCs and TSCs (Fig. 5a), was substantially hypermethylated in sperm of F0 $M t r r^{+/ g t}$ males compared with controls $(39.0 \pm 4.1 \%$ more methylated CpGs per CpG site assessed; Fig. 5b, c). As with the other sperm DMRs assessed (Fig. 3), the Hira DMR was reprogrammed in F1-F3 wildtype embryos and placentas at E10.5 (Fig. 3a, Supplementary Fig. 11a). Although we originally assessed Hira mRNA in the F1-F2 generations in the maternal grandfather pedigree (Fig. 4), further analysis revealed that Hira isoforms (mRNA and long non-coding RNA (lncRNA 209); Fig. 5a) were differentially regulated at E10.5 (Fig. 6a-c). Hira lncRNA function is unknown, though lncRNA-based mechanisms often control cell fates during development by influencing the nuclear organisation and transcriptional regulation ${ }^{53}$. Notably, this pattern of RNA expression was associated with generational patterns of phenotypic inheritance. For instance, we observed down-regulation of Hira mRNA in F2-F3 wildtype embryos and not F1 wildtype embryos at E10.5 (Fig. 6a-c). Conversely, significant upregulation of Hira lncRNA expression was apparent only in F1 wildtype embryos at E10.5 (Fig. 6a-c). This expression pattern was embryo-specific since the corresponding placentas showed normal Hira transcript levels in each generation assessed compared to controls (Fig. 6a-b). Since phenotypes at E10.5 were apparent in F2 generation onwards, yet were absent in the F1 generation of the $\mathrm{Mtrr}^{+/ g t}$ maternal grandfather pedigree ${ }^{3}$, embryo-specific Hira RNA misexpression reflected the pattern of phenotypic inheritance.

To further investigate a potential link between Hira expression and phenotypic inheritance, we analysed wildtype F1-F3 conceptuses at E10.5 derived from F0 $\mathrm{Mtrr}^{+/ g t}$ females (i.e., the maternal grandmother pedigree; Supplementary Fig. 1e). The breeding scheme was as follows: F0 Mtrr ${ }^{+/ g t}$ females were mated with $\mathrm{C} 57 \mathrm{Bl} / 6 \mathrm{~J}$ control males. The F1 progeny was collected at E10.5 for genotype and phenotype analysis or allowed to litter out. The resulting adult F1 wildtype females were mated with C57Bl/6 J males. The F2 wildtype progeny was similarly collected at E10.5 for analysis or adult F2 wildtype females were mated with $\mathrm{C} 57 \mathrm{Bl} / 6 \mathrm{~J}$ control males to generate F3 wildtype conceptuses for analysis at E10.5 (Supplementary Fig. 1e). In this pedigree, all generations, including the F1 generation, display a broad spectrum of developmental phenotypes ${ }^{3}$. Supporting our hypothesis, Hira mRNA expression was downregulated in F1-F3 wildtype embryos derived from an F0 $\mathrm{Mtrr}^{+/ g t}$ female compared to $\mathrm{C} 57 \mathrm{Bl} / 6 \mathrm{~J}$ controls (Fig. 6d), thus correlating with maternal 


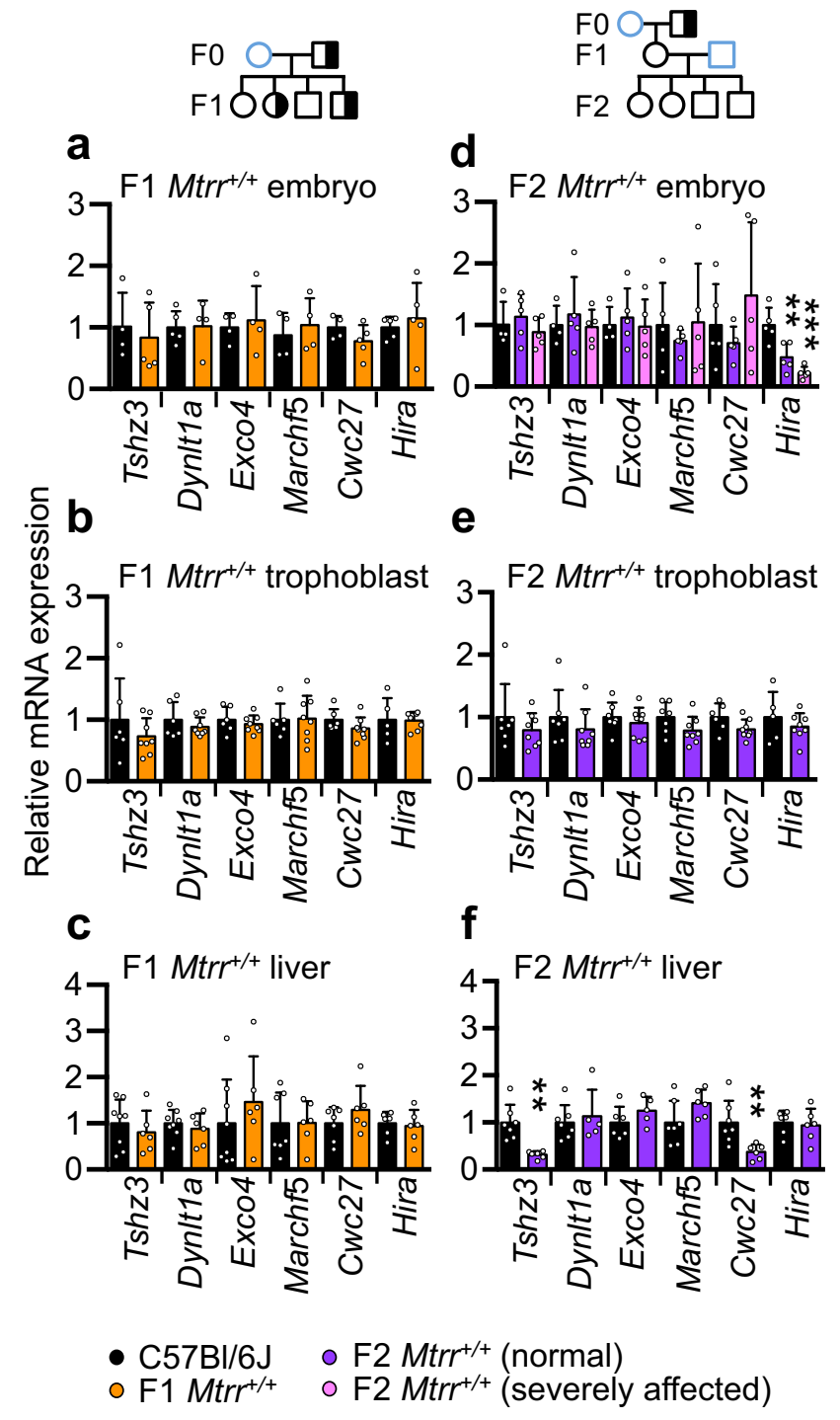

Fig. 4 Transcriptional changes associated with some DMR-associated genes in $\mathbf{F 1}$ and $\mathbf{F 2}$ wildtype somatic tissue. a-f RT- $\mathrm{aPCR}$ analysis of mRNA expression of genes located in close proximity to sperm DMRs in a, d embryos at E10.5, b, e placental trophoblast at E10.5, and $\mathbf{c}$, $\mathbf{f}$ adult livers of wildtype $\left(\mathrm{Mtrr}^{+/+}\right) \mathrm{F} 1$ and F2 progeny. Tissue from phenotypically normal F1 wildtype individuals (orange bars) or F2 wildtype individuals (purple bars) or severely affected F2 wildtype individuals (pink bars) derived from an FO $\mathrm{Mtrr}^{+/ \mathrm{gt}}$ male was assessed. C57BI/6 J control tissues (black bars) were assessed as controls. Pedigrees indicate specific mating schemes assessed (see also Supplementary Fig. 1a, d). Data are plotted as mean \pm standard deviation, and are presented as relative expression to $\mathrm{C} 57 \mathrm{BI} / 6 \mathrm{~J}$ levels (normalised to 1). Embryos: $\mathrm{C} 57 \mathrm{Bl} / 6 \mathrm{~J}, \mathrm{~N}=4-5$ embryos; $\mathrm{F} 1 \mathrm{Mtrr}+/+, \mathrm{N}=4-5$ embryos; F2 Mtrr ${ }^{+/+}$phenotypically normal or severely affected, $N=5$ embryos. Trophoblast: $\mathrm{C} 57 \mathrm{Bl} / 6 \mathrm{~J}, \mathrm{~N}=5-7$ placentas; F1 or F2 $\mathrm{Mtrr}+/+$, $N=8$ placentas. Liver: $\mathrm{C} 57 \mathrm{BI} / 6 \mathrm{~J}, \mathrm{~N}=6-8$ livers; $\mathrm{F} 1 \mathrm{Mtrr}+/+, N=6$ livers; F2 $M \mathrm{trr}^{+/+}, N=5-6$ livers. Independent $t$ tests or one-way ANOVA with Dunnett's multiple comparisons tests were performed. d Hira ${ }^{\star \star} p=$ $0.0049,{ }^{* \star *} p=0.0002 ; \mathbf{f} T$ shz3 ${ }^{\star *} p=0.0011 ;$ Cwc27 ${ }^{* \star} p=0.0095$. Each gene was associated with the following sperm DMR shown in Fig. 3: Tshz3, DMR E115; Dynlt1a, DMR E52; Exoc4, DMR E112; March5, DMR C7; Cwc27, DMR D20; Hira, DMR A10. F0, parental generation; F1 first filial generation; F2, second filial generation. Pedigree legend: circle, female; square, male; blue outline, C57BI/6 J control; black outline, Mtrrgt mouse line; white filled, Mtrr ${ }^{+/+}$; half-white/half-black filled, $\mathrm{Mtrr}^{+/ g t}$. Source data are provided as a Source Data file. phenotypic inheritance. As expected, Hira lncRNA transcripts were unchanged in F1 and F3 wildtype embryos (Fig. 6d). However, Hira lncRNA was downregulated in F2 wildtype embryos (Fig. 6d), which display the highest frequency of phenotypes among the three generations ${ }^{3}$. Regardless, these data suggested that altered Hira mRNA transcripts might be a potential biomarker of maternal phenotypic inheritance since dysregulation of Hira mRNA occurred only in wildtype embryos with high phenotypic risk as a result of their derivation from an oocyte with Mtrrgt ancestry rather than sperm (Fig. 7).

How the Hira gene is regulated is unknown. Mtrrst/gt embryos at E10.5 (derived from Mtrrst/gt intercrosses), which demonstrate a greater phenotypic risk than the $M t r r^{+/ g t}$ maternal grandparental pedigrees ${ }^{3}$, also displayed dysregulation of Hira mRNA and lncRNA expression (Supplementary Figs. 8k-m, 11d). This finding was in association with normal DNA methylation at the Hira DMR in Mtrr ${ }^{g t / g t}$ embryos at E10.5 (Supplementary Fig. 8b), implicating additional mechanisms of epigenetic regulation. Histone methylation profiles at the Hira locus in normal ESCs and TSCs indicate a potential role for the Hira promoter and Hira DMR (Fig. 5a) in gene regulation that will require future investigation.

HIRA protein levels were also dysregulated in $M t r r^{g t} / g^{t}$ embryos and F2 wildtype embryos and placentas (Fig. 6e-h, Supplementary Fig. 11e, f). The pattern of dysregulation did not always occur in a manner predicted by the direction of Hira mRNA expression. For example, there was an increase in HIRA protein levels in F2 wildtype embryos when Hira mRNA was downregulated (Fig. 6b, d-h). This discrepancy might result from defective HIRA protein degradation, drastic translational upregulation of HIRA protein to compensate for low mRNA levels, or alternatively, negative feedback to down-regulate Hira mRNA owing to high levels of HIRA protein in the embryo. Further work will be required to delineate whether the HIRA chaperone mediates maternal phenotypic inheritance in the Mtrrgt model and other models of TEI.

\section{Discussion}

We investigated potential mechanisms contributing to epigenetic inheritance in Mtrrgt mice, a unique model of mammalian $\mathrm{TEI}^{3}$. In the Mtrrst model, inheritance of developmental phenotypes and epigenetic instability occurs via the maternal grandparental lineage with an F0 Mtrr ${ }^{+/ g t}$ male or female initiating the TEI effect $^{3}$. Here, we assessed DNA methylation in spermatozoa to understand how the germline epigenome was affected by the $M t_{r}{ }^{g t}$ allele. We chose sperm due to its experimental tractability and to avoid the confounding factors of the F0 uterine environment when assessing epigenetic inheritance via the germline. We identified several distinct DMRs in regions of predicted importance in transcriptional regulation and epigenetic inheritance including regions of nucleosome retention and reprogramming resistance. This result illustrates widespread epigenetic instability in the male germline of the Mtrrot model, particularly in the F0 $M t r r^{+/ g t}$ males of the maternal grandfather pedigree. While largely resolved in somatic tissue of subsequent wildtype generations, some germline DMRs were correlated with transcriptional changes at associated loci in the F1-F3 progeny. This proposed transcriptional memory of a germline DMR persisted for at least three generations, longer than previously reported in another model $^{4}$. This observation indicates additional epigenetic factors beyond DNA methylation in the mechanism of TEI. Furthermore, the histone chaperone gene Hira emerged as a transcriptional biomarker and potential mediator of maternal phenotypic inheritance. 


\section{a}

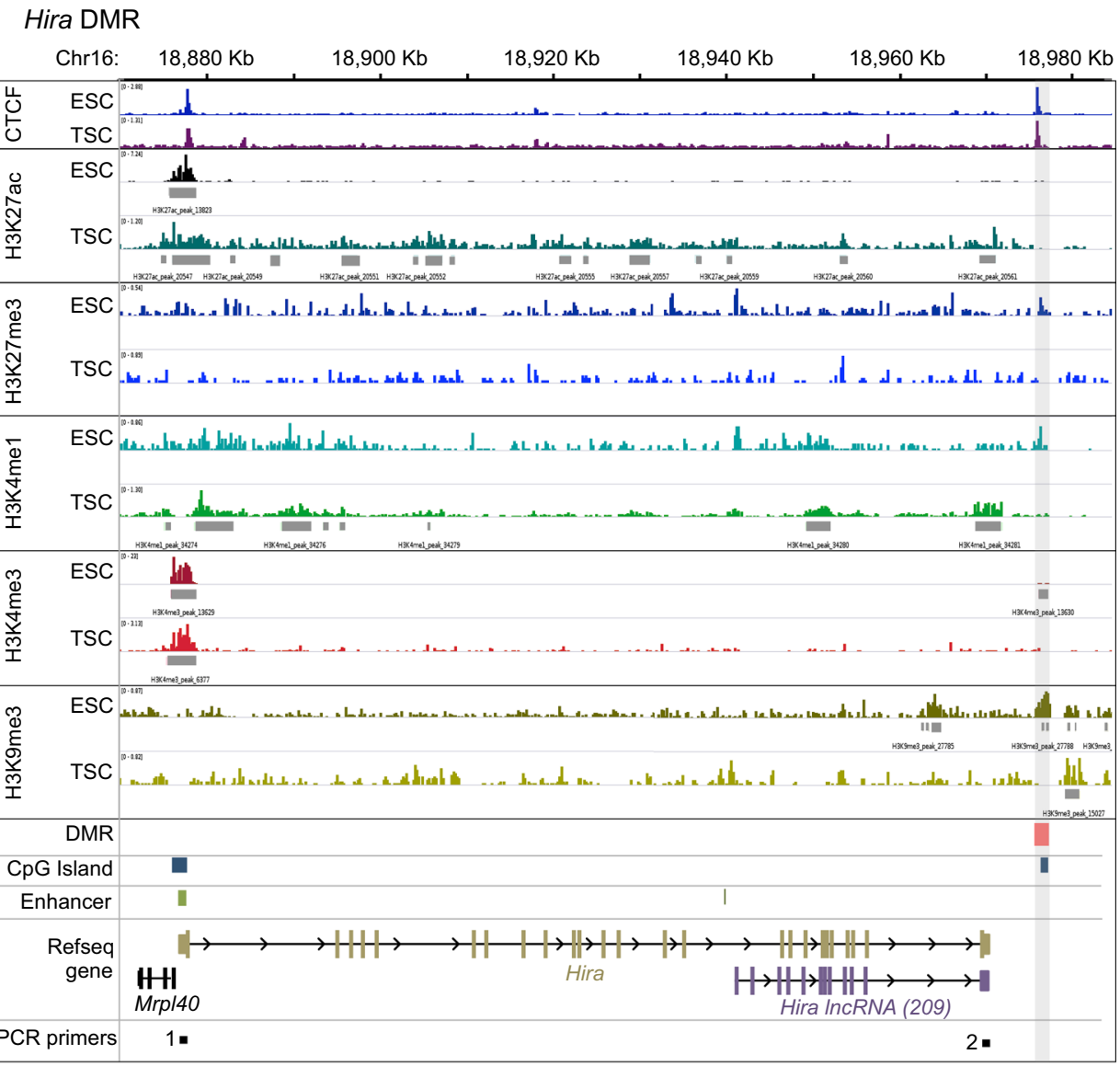

b MeDIP-seq track (sperm)

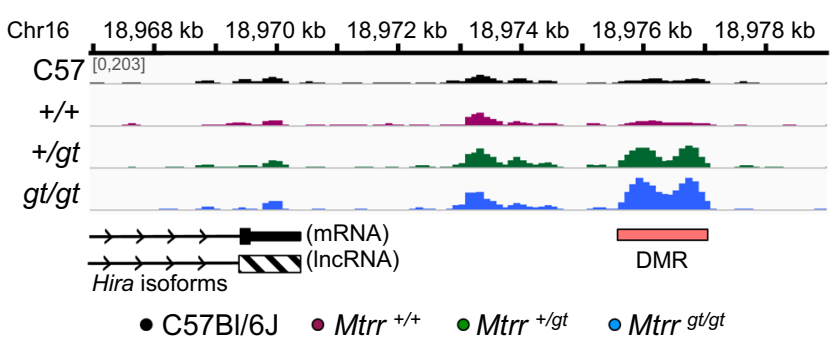

C

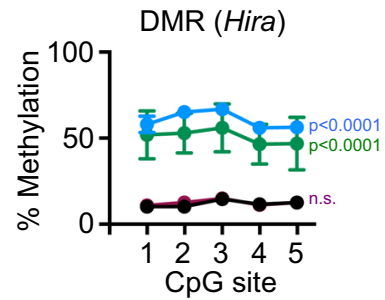

Fig. 5 Epigenetic characteristics of Hira DMR in mice. a Enrichment of DNA binding proteins (CCTC-binding factor, CTCF) and histone modifications (H3K27ac, H3K27me3, H3K4me1, H3K4me3, H3K9me3) in the Hira locus on mouse chromosome (Chr) 16 using published data sets ${ }^{35}$ of chromatin immunoprecipitation followed by sequencing (ChIP-seq) analyses in cultured wildtype embryonic stem cells (ESCs) and trophoblast stem cells (TSCs). Dark grey rectangles indicate enrichment peak calling for each histone modification. Pink rectangle and light grey shading indicate the Hira differentially methylated region (DMR) identified in sperm of $\mathrm{Mtrr}^{+/ g t}$ and $M t r r^{g t / g t}$ males. Blue rectangles indicate $\mathrm{CpG}$ islands. Green rectangles indicate enhancers. Schematics of protein-encoding (brown) and long non-coding RNA (IncRNA) encoding (purple) Hira isoforms are shown. Region of qPCR primer sets 1 and 2 are also indicated (black boxes). b Partial schematic drawing of Hira transcripts and Hira DMR (pink rectangle) in relation to MeDIP-seq reads in sperm from C57BI/6 J (black), wildtype (Mtrr ${ }^{+/}$; purple), Mtrr ${ }^{+/ g t}$ (green) and Mtrgt/gt (blue) males. $N=8$ males/group. c The average percentage methylation at individual $\mathrm{CpG}$ sites (mean \pm standard deviation) in the Hira DMR in sperm from C57Bl/6 J males (black circles; $N=4$ males), Mtrr $+/+$ males (purple circles; $N=4$ males), $M t r{ }^{+} / g t$ males (green circles; $N=8$ males) and $M t r r g t / g t$ males(blue circles; $N=8$ males). Two-way ANOVA with Sidak's multiple comparisons test performed on mean methylation per $\mathrm{CpG}$ site. $p$ values are indicated for each male genotype compared to $\mathrm{C} 57 \mathrm{Bl} / 6 \mathrm{~J}$ and were similar for all CpG sites assessed within one genotype as indicated: $\mathrm{C} 57 \mathrm{BI} / 6 \mathrm{~J}$ versus $\mathrm{Mtrr}+/+$, not significant (n.s.); C57BI/6 J versus Mtrr ${ }^{+/ g t}$ males or Mtrrgt/gt males, $p<0.0001$. Source data are provided as a Source Data file.

The extent to which genetic and epigenetic factors interact in this and other TEI models is unclear. One-carbon metabolism is involved in thymidine synthesis ${ }^{54}$, and DNA breaks triggered by folate deficiency-induced uracil misincorporation were demonstrated in erythrocytes of splenectomised patients ${ }^{55}$ and prostate adenoma cells ${ }^{56}$. However, WGS of $M t_{r}$ st/gt $^{g t}$ embryos excluded genetic instability in the Mtrrst mouse line because de novo mutations occurred at an expected ${ }^{28}$ and comparable frequency to control embryos. The WGS data also discounted alternative phenotype-causing mutations outside of the Mtrr locus and, when compared with our sperm methylome data set, showed that differential CpG methylation was unlikely due to underlying genetic variation in the Mtrrgt mouse line. Therefore, the epigenetic consequences of the Mtrrot allele rather than genetic 
a

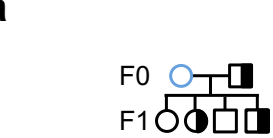

b

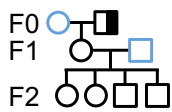

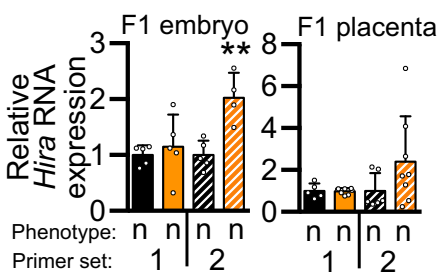

F2 embryo
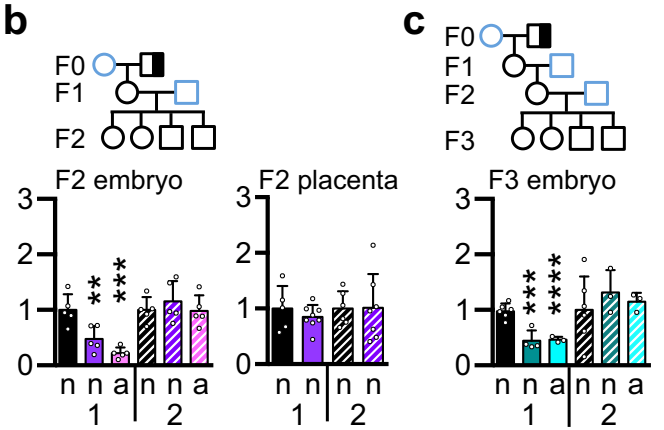

d
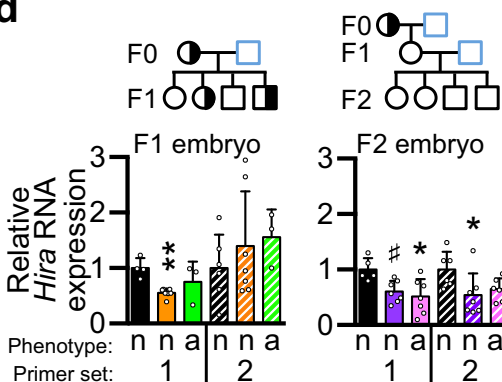

$37^{\mathrm{F} 2}$ embryo

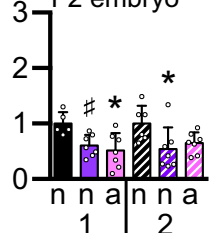

e
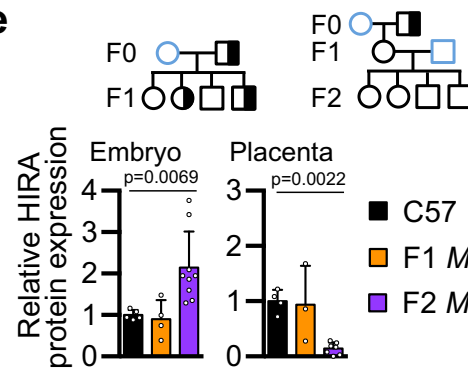

Placenta

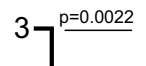

- $\mathrm{C} 57$

口 F1 Mtrrt+t

F2 $\mathrm{Mtrr}^{+/+}$

f

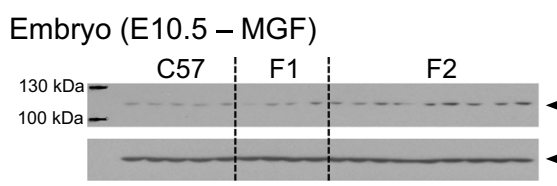

Placenta (E10.5 - MGF)

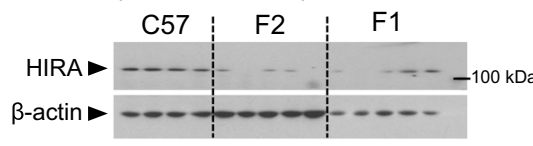

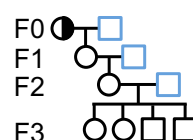

$47^{\mathrm{F} 3}$ embryo

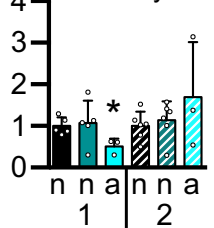

g
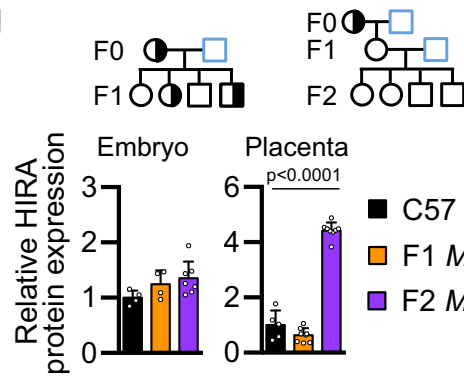

Placenta

- C57BI/6J

$\square$ F1 Mtrrt/+ (normal [n])

口 F1 Mtrrt+t

(severely affected [a])

$\square$ F2 $\mathrm{Mtrr}^{+/+}(\mathrm{n})$

F2 $\mathrm{Mtrr}^{+/+}$(a)

F3 $\mathrm{Mtrr}^{+/+}$(n)

F3 $\mathrm{Mtrr}^{+/+}$(a)

h

Embryo (E10.5 - MGM)

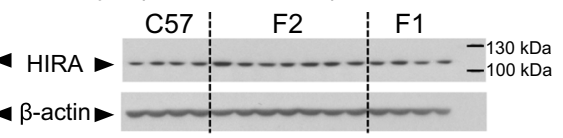

Placenta (E10.5 - MGM)

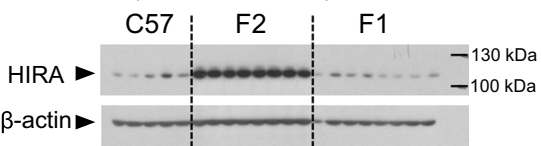

instability are more likely to instigate TEI in this model. Generating alternative Mtrr mutations and/or backcrossing the Mtrrst allele into a different mouse strain will further assess whether genetics has a role in TEI.

Our data show that inheritance of sperm DMRs by offspring somatic tissue was unlikely, as have other studies ${ }^{4,49,50}$. Instead, somatic cell lineages might inherit germline epigenetic instability in a broader sense. For instance, despite normal DNA methylation in F1-F3 wildtype embryos and placentas (E10.5) at the genomic locations highlighted by sperm DMRs, epigenetic instability associated with gene misexpression is still evident in F1 and F2 wildtype placentas at several loci ${ }^{3}$. It is possible that abnormalities in the sperm epigenome of F0 Mtrr $/ g t$ males might be reprogrammed and then stochastically and abnormally reestablished/maintained in other genomic regions in wildtype offspring. This hypothesis might explain inter-individual phenotypic variability in the F2-F3 generations. However, we showed that sperm of $M t r r+/+$ males exhibited several DMRs that overlapped with sperm DMRs in $M t r r^{+/ g t}$ males (representing their fathers). Therefore, reconstruction of specific atypical F0 germline methylation patterns in the F1 wildtype germline is possible in the Mtrr ${ }^{\text {t }}$ model. Vinclozolin toxicant exposure model of TEI shows dissimilar DMRs in spermatozoa of F1 and F3 offspring 8 , which suggests that epigenetic patterns might shift as the generational distance from the F0 individual increases.

Dietary folate deficiency causes differential methylation in sperm and craniofacial defects in the immediate offspring 49 , though whether it leads to TEI is unknown. There was no overlap of DMRs when sperm methylomes were compared between the diet model and $M t r r^{+/ g t}$ males. This result disputes the existence of folate-sensitive epigenomic hotspots in sperm. However, the severity of insult or technical differences (e.g., MeDIP-array ${ }^{49}$ versus MeDIP-seq) might explain the discrepancy. Why only one Mtrrst allele sufficiently initiates TEI is unknown since a direct 
Fig. 6 Dysregulation of Hira RNA expression in embryos aligns with a pattern of maternal phenotypic inheritance. a-d RT-qPCR analysis of Hira mRNA (solid bars, primer set 1) and Hira IncRNA (striped bars, primer set 2) expression in embryos and placentas at E10.5. The tissue and pedigrees were assessed (see also Supplementary Fig. 1a, d-e for the breeding scheme): a F1 wildtype $\left(\mathrm{Mtrr}^{+/+}+\right.$) conceptuses from FO Mtrr $+/ g t$ males (orange bars; $\mathrm{N}=$ 4-5 embryos, $N=8$ placentas), b F2 wildtype conceptuses from FO Mtrr $+/ g t$ males (purple bars, $N=5$ embryos and $N=7-8$ placentas from phenotypically normal ( $n$ ) conceptuses; pink bars, $N=5$ severely affected (a) embryos), c F3 wildtype conceptuses from FO Mtrr $+/ g t$ males (teal bars, $N=$ 3-4 phenotypically normal embryos; turquoise bars; $N=3$ severely affected embryos), and $\mathbf{d}$ F1 wildtype embryos from FO Mtrr $+/ g t$ females (orange bars, $N=7$ phenotypically normal embryos; green bars, $N=3$ severely affected embryos), F2 wildtype embryos from FO Mtrr $+/ g t$ females (purple bars, $N=7$ phenotypically normal embryos; pink bars, $N=7$ severely affected embryos), and F3 wildtype embryos from F0 Mtrr $+/ g t$ females (teal bars, $N=5-7$ phenotypically normal embryos; turquoise bars, $N=3$ severely affected embryos). C57BI/6 $\mathrm{J}$ conceptuses were controls (black bars, $N=4-8$ embryos or placenta/experiment). e-h Western blot analysis showing HIRA protein expression in F1 wildtype (orange bars) and F2 wildtype (purple bars) conceptuses derived from e to f FO Mtrr ${ }^{+/ g t}$ males or $\mathbf{g}-\mathbf{h}$ FO Mtrr ${ }^{+/ g t}$ females. C57BI/6 J (C57) conceptuses (black bars) were assessed as controls. e, $\mathbf{f}$ C57BI6/J: $N=$ 4-5 embryos, $N=4-5$ placentas; F1: $N=4$ embryos, $N=3-8$ placentas; F2: $N=7-9$ embryos, $N=7-8$ placentas. Images of western blot gels showing HIRA protein with $\beta$-actin as a loading control in $\mathbf{f}$ and $\mathbf{h}$ were quantified in $\mathbf{e}$ and $\mathbf{g}$, respectively, using the background subtraction method. HIRA protein levels were normalised to $\beta$-actin. All RNA and protein data were plotted as mean \pm standard deviation and relative to C57BI/6 J (normalised to 1). Experiments were conducted in technical duplicates (protein) or triplicates (RNA). Statistical analyses: a-d Two-tailed independent $t$ test or Kruskal-Wallis test with Dunn's multiple comparison. $\mathbf{a}{ }^{\star \star} p=0.0034 ; \mathbf{b}{ }^{\star \star} p=0.0049,{ }^{\star \star \star} p=0.0002 ; \mathbf{c}^{\star \star \star} p=0.0004,{ }^{\star \star \star \star} p<0.0001 ; \mathbf{d ~ F 1 : ~}{ }^{\star \star} p=0.0056, \mathrm{~F} 2$ (primer set 1): ${ }^{\#} p=0.06,{ }^{\star} p=0.0203$, F2 (primer set 2): ${ }^{\star} p=0.0457, F 3:{ }^{\star} p=0.0161$. e, f One-way ANOVA. $p$ values are shown on graphs. Pedigree legend: circle, female; square, male; blue outline, C57BI/6 J line; black outline, Mtrrgt mouse line; white fill, Mtrr ${ }^{+/+}$; half-black-half-white fill, Mtrr ${ }^{+/ g t}$; black fill, Mtrrgt/gt . F0, parental generation; F1, first filial generation; F2, second filial generation; F3, third filial generation. Source data are provided as a Source Data file.
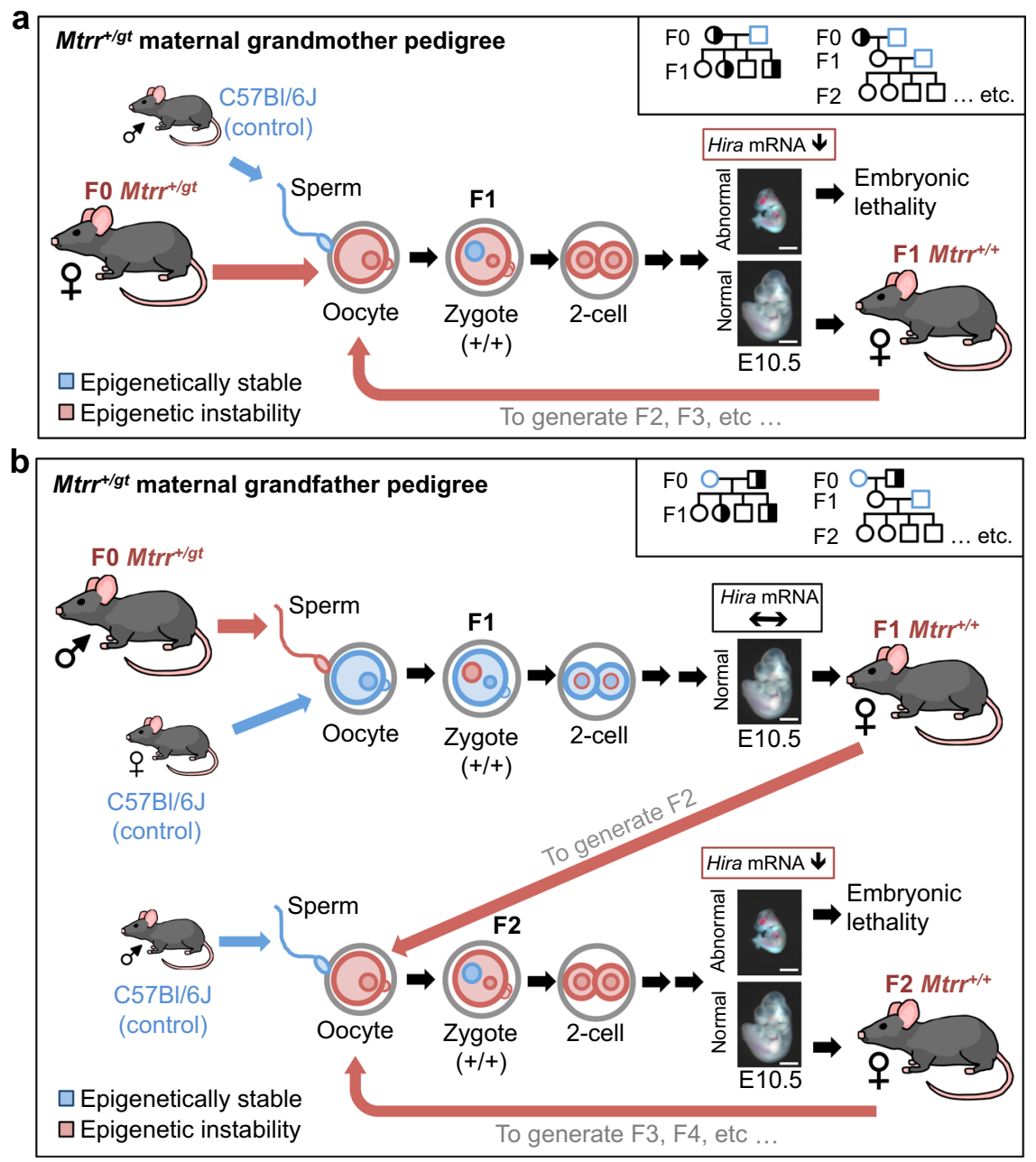

Fig. 7 Proposed model of maternal grandparental phenotype inheritance in Mtrrgt model that implicates epigenetic instability in the germline. A model proposing how epigenetic instability generated by the Mtrrgt mutation might be differently inherited over multiple generations depending upon whether TEI is initiated by $\mathbf{a}$ an oocyte or $\mathbf{b}$ sperm of an FO Mtrr + gt individual. Trends of Hira mRNA expression are shown. Pedigree legend: circle, female; square, male; blue outline, C57BI/6 J control; black outline, Mtrrgt mouse line; white filled, Mtrr ${ }^{+/+}$; half-white/half-black filled, Mtrr ${ }^{+/ g t}$. See also Supplementary Fig. 1 for breeding schemes. Scale bar: $500 \mu \mathrm{m}$. FO, parental generation; F1, first filial generation; F2, second filial generation; F3, third filial generation. 
paramutation effect was not evident ${ }^{3}$ and $\mathrm{Mtrr}^{+/ g t}$ mice do not display similar metabolic derangement to $\mathrm{Mtrr}^{\mathrm{gt} / g t}$ mice $\mathrm{e}^{3,12}$.

Whether specific DNA methylation patterns observed in F0 sperm of the maternal grandfather pedigree are reconstructed ${ }^{57,58}$ in F1 oocytes is yet-to-be determined. Extensive differences between sperm and oocytes methylomes ${ }^{59}$ will make this difficult to resolve and yet also emphasizes that additional epigenetic mechanisms are likely involved, such as histone modifications ${ }^{10}$, sncRNA expression ${ }^{1,9}$ and/or changes in nucleosome composition and spacing that alter nuclear architecture ${ }^{59}$. Several of these mechanisms implicate regulators like HIRA ${ }^{25-27}$ (see below). Future studies will explore the extent to which differential epigenetic marks in oocytes from F0 $\mathrm{Mtrr}^{+/ g t}$ females are reconstructed in oocytes of subsequent generations, through embryo transfer experiments will be required to exclude the confounding effects of the F0 uterine environment ${ }^{60}$.

Several recent studies have assessed sncRNA expression (e.g., microRNA or tRNA fragments [tsRNA] $)^{1,2,9}$ in sperm as a mechanism in the direct epigenetic inheritance of disease. For example, manipulating paternal diet in mice is sufficient to alter tsRNA content in spermatozoa leading to altered expression of genes associated with MERVL elements in early F1 embryos ${ }^{9}$ and metabolic disease in $\mathrm{F} 1$ adult offspring ${ }^{1}$. It is possible that sncRNAs in sperm from F0 Mtrr ${ }^{+/ g t}$ males are misexpressed and/ or abnormally modified ${ }^{61}$, and that this might contribute to TEI mechanisms. Although it is currently unclear how sperm ncRNA content causes phenotypic inheritance beyond the F1 generation, genes involved in the development of the primordial germ cell population within the F1 wildtype female embryo might be among those affected. Exploring sncRNA content in germ cells of the Mtrrgt model will help to better understand this question.

Several sperm DMRs in $\mathrm{Mtrr}^{+/ g t}$ males (e.g., Cwc27, Tshz3, Hira) were associated with transcriptional changes in F2-F3 wildtype embryos and adult liver. We focused our analysis on the Hira DMR due to its responsiveness to the severity of the Mtrrgt genotype, the fact that $\mathrm{Hira}^{-1-}$ embryos $^{52}$ phenocopy embryos in the Mtrrgt model $^{3}$, and because the potential consequences of HIRA dysfunction are multifaceted and implicated in epigenetic inheritance. The HIRA histone chaperone complex regulates histone deposition/recycling required to maintain chromatin integrity in the oocyte and zygote $\mathrm{e}^{25,26,62}$. Additionally, HIRA is implicated in rRNA transcription ${ }^{27}$. Therefore, dysregulation of HIRA expression and/or function in the Mtrrst model might alter nucleosome spacing and ribosome heterogeneity ${ }^{63,64}$ with substantial implications for regulation of transcriptional pathways during germ cell and embryo development.

Since the F1 progeny differ phenotypically when derived from an F0 $\mathrm{Mtrr}^{+/ \mathrm{gt}}$ male versus F0 Mtrr ${ }^{+/ \mathrm{gt}} \mathrm{female}^{3}$, the mechanism initiating TEI potentially differs between sperm and oocytes. The extent of mechanistic overlap is not well understood. Certainly, paternally inherited epigenetic factors ${ }^{1,2,4,9,10,49,61,65}$ are better studied than maternally-inherited factors ${ }^{60,66}$. The difference in phenotypic severity in the Mtrrot model might relate to a cytoplasmically inherited factor in the F1 wildtype zygote (Fig. 7). HIRA is a suitable candidate since maternal HIRA ${ }^{25,67}$ is involved in protamine replacement by histones in the paternal pronucleus ${ }^{27}$. Therefore, abnormal HIRA expression or function in oocytes would potentially perpetuate epigenetic instability in the next generation. In the case of the Mtrrgt model, dysregulation of Hira and/or other maternal factors in oocytes might have a greater impact on epigenetic integrity in the early embryo than when dysregulated in sperm.

Overall, we show the potential long-term transcriptional and phenotypic impact of abnormal folate metabolism on germline DNA methylation and emphasise the complexity of epigenetic mechanisms involved in TEI. Ultimately, our data indicate the importance of normal folate metabolism in both women and men of reproductive age for healthy pregnancies in their daughters and granddaughters.

\section{Methods}

Ethics statement. This research was regulated under the Animals (Scientific Procedures) Act 1986 Amendment Regulations 2012 following ethical review by the University of Cambridge Animal Welfare and Ethical Review Body.

Animal model. MtrrGt(XG334)Byg (MGI:3526159) mouse line, referred to as the Mtrrst model, was generated when a $\beta$-geo gene-trap (gt) vector was inserted into intron 9 of the Mtrr gene in 129P2Ola/Hsd ESCs ${ }^{3}$. Mtrr ${ }^{g t}$ ESCs were injected into $\mathrm{C} 57 \mathrm{Bl} / 6 \mathrm{~J}$ blastocysts. Upon germline transmission, the Mtrrgt allele was backcrossed into the $\mathrm{C} 57 \mathrm{Bl} / 6 \mathrm{~J}$ genetic background for at least eight generations ${ }^{3}$. $\mathrm{Mtrr}^{+/+}$and $\mathrm{Mtrr}^{+/ g t}$ mice were produced from $\mathrm{Mtrr}^{+/ g t}$ intercrosses (Supplementary Fig. 1b). Mtrrgt/gt mice were produced from $M t r r^{g t / g t}$ intercrosses (Supplementary Fig. 1c). C57Bl/6 J mice from The Jackson Laboratories (www.jaxmice. jax.org) and 129P2Ola/Hsd from Envigo (previously Harlan Laboratories [www. envigo.com]) were used as controls and were bred in house and separately from the $M$ trr $^{g t}$ mouse line. Mice were housed in a temperature- and humidity-controlled environment with a $12 \mathrm{~h}$ light-dark cycle. All mice were fed a normal chow diet (Rodent No. 3 chow, Special Diet Services) ad libitum from weaning, which included (per $\mathrm{kg}$ of diet): $1.6 \mathrm{~g}$ choline, $2.73 \mathrm{mg}$ folic acid, $26.8 \mu \mathrm{g}$ vitamin $\mathrm{B}_{12}, 3.4 \mathrm{~g}$ methionine, $51.3 \mathrm{mg}$ zinc. Mice were killed via cervical dislocation. Genotyping for the $\mathrm{Mtrr}^{+}$and $\mathrm{Mtrr}^{g t}$ alleles and for sex $(\mathrm{Rbm} 31)$ was performed ${ }^{3,68,69}$ using PCR on DNA extracted from ear tissue or yolk sac using primer sequences in Supplementary Table 8 .

To determine the multigenerational effects of the Mtrrgt allele in the maternal grandfather, the following mouse pedigree was established (Supplementary Fig. 1d). For the F1 generation, F0 Mtrr ${ }^{+/ g t}$ males were mated with C57Bl/6 J females and the resulting $M t r r^{+/+}$progeny were analysed. For the F2 generation, F1 Mtrr ${ }^{+/+}$females were mated with $\mathrm{C} 57 \mathrm{Bl} / 6 \mathrm{~J}$ males and the resulting Mtrr ${ }^{+/+}$ progeny were analysed. For the F3 generation, F2 Mtrr ${ }^{+/+}$females were mated with $\mathrm{C} 57 \mathrm{Bl} / 6 \mathrm{~J}$ males and the resulting $\mathrm{Mtrr}+/+$ progeny was analysed. A similar pedigree was established to assess the effects of the Mtrrgt allele in the maternal grandmother with the exception of the F0 generation, which involved the mating of an $M t r r^{+/ g t}$ female with a C57Bl/6 J male (Supplementary Fig. 1e).

Tissue dissection and phenotyping. For embryo and placenta collection, timed matings were established and noon on the day that the vaginal plug was detected was considered embryonic day (E) 0.5 . Embryos and placentas were dissected at E10.5 in cold $1 \times$ phosphate-buffered saline and were scored for phenotypes (see below), photographed, weighed, and snap frozen in liquid nitrogen for storage at $-80^{\circ} \mathrm{C}$. All conceptuses were dissected using a Zeiss SteREO Discovery V8 microscope with an AxioCam MRc5 camera (Carl Zeiss). Livers were collected from pregnant female mice (gestational day 10.5), weighed and snap frozen in liquid nitrogen for storage at $-80^{\circ} \mathrm{C}$. Both male and female conceptuses at E10.5 were assessed, as no sexual dimorphism is apparent at this stage $\mathrm{f}^{70}$. While many individual mice were assessed over the course of this study, some of the individual tissue samples were assessed for the expression of multiple genes or for methylation at multiple DMRs.

A rigorous phenotyping regime was performed at E10.5 as previously described ${ }^{3}$. In brief, all conceptuses were scored for one or more congenital malformation (as appropriate for the developmental stage) including failure of the neural tube to close in the cranial or spinal cord region, malformed branchial arches, pericardial edema, reversed heart looping, enlarged heart, and/or eccentric chorioallantoic attachment Twinning or haemorrhaging was also scored as a severe abnormality. Conceptuses with severe abnormalities were categorised separately from resorptions, the latter of which consisted of maternal decidua surrounding an indistinguishable mass of fetally derived tissue. Resorptions were not assessed in this study as they represented dead conceptuses. Embryos with $<30$ somite pairs were considered developmentally delayed Embryos with 30-39 somite pairs but a crown-rump length more than two standard deviations (sd) from the mean crown-rump length of $\mathrm{C} 57 \mathrm{Bl} / 6 \mathrm{~J}$ control embryos were considered growth restricted or growth enhanced. Conceptuses were considered phenotypically normal if they were absent of congenital malformations, had 30-39 somite pairs, and had crown-rump lengths within two sd of controls. AxioVision 4.7.2 imaging software was used to measure crown-rump lengths (Carl Zeiss). Conceptus size at E10.5 was unaffected by litter size in all Mtrrgt pedigrees assessed ${ }^{70}$.

Spermatozoa collection. Spermatozoa from cauda epididymides and vas deferens were collected from 16 to 20 week-old fertile mice using a swim-up procedure as previously described ${ }^{38}$ with the following amendments. Spermatozoa were released for $20 \mathrm{~min}$ at $37^{\circ} \mathrm{C}$ in Donners Medium ( $25 \mathrm{mM} \mathrm{NaHCO}, 20 \mathrm{mg} / \mathrm{ml}$ bovine serum albumin, $1 \mathrm{mM}$ sodium pyruvate and $0.53 \%$ (vol/vol) sodium dl-lactate in Donners stock ( $135 \mathrm{mM} \mathrm{NaCl}, 5 \mathrm{mM} \mathrm{KCl}, 1 \mathrm{mM} \mathrm{MgSO} 4,2 \mathrm{mM} \mathrm{CaCl}_{2}$ and $30 \mathrm{mM}$ HEPES)). Samples were centrifuged at $500 \times g\left(21^{\circ} \mathrm{C}\right)$ for $10 \mathrm{~min}$. The supernatant was transferred and centrifuged at $1300 \times g\left(4^{\circ} \mathrm{C}\right)$ for $15 \mathrm{~min}$. After the majority of 
supernatant was discarded, the samples were centrifuged at $1300 \times g\left(4^{\circ} \mathrm{C}\right)$ for $5 \mathrm{~min}$. Further supernatant was discarded and the remaining spermatozoa were centrifuged at $12,000 \times g$ for $1 \mathrm{~min}$ and stored at $-80^{\circ} \mathrm{C}$.

Nucleic acid extraction. For embryo, trophoblast and liver tissue, genomic DNA (gDNA) was extracted using DNeasy Blood and Tissue kit (Qiagen) according to the manufacturer's instructions. RNA was extracted from tissues using the AllPrep DNA/RNA Mini Kit (Qiagen). For sperm, Solution A (75 mM NaCl pH 8; $25 \mathrm{mM}$ EDTA) and Solution B (10 mM Tris- $\mathrm{HCl} \mathrm{pH} 8 ; 10 \mathrm{mM}$ EDTA; $1 \%$ SDS; $80 \mathrm{mM}$ DTT) were added to the samples followed by RNAse A incubation $\left(37^{\circ} \mathrm{C}, 1 \mathrm{~h}\right)$ and Proteinase $\mathrm{K}$ incubation $\left(55^{\circ} \mathrm{C}\right.$, overnight) as was previously described ${ }^{4}$. DNA was extracted using phenol/chloroform/isoamyl alcohol mix (25:24:1) (Sigma-Aldrich) as per the manufacturer's instructions and pelleted using standard methods in TE buffer. DNA quality and quantity were confirmed using gel electrophoresis and QuantiFluor dsDNA Sample kit (Promega) as per the manufacturer's instructions.

Whole-genome sequencing. Non-degraded gDNA from two whole C57Bl/6 J embryos at E10.5 (one male, one female) and six whole Mtrrgt/gt embryos with congenital malformations at E10.5 (four males, two females) derived from Mtrrgt/gt intercrosses (Supplementary Fig. 1a, c) was sent to BGI (Hong Kong) for library preparation and sequencing. The libraries of each embryo were sequenced separately. Sequencing was performed with $150 \mathrm{bp}$ paired-end reads on an Illumina HiSeq X machine. Quality control of reads assessed with FastQC (version 0.11.5, http://www. bioinformatics.babraham.ac.uk/projects/fastqc/). Adaptors and low-quality bases removed using Trim Galore (version 0.6.4, https://www.bioinformatics.babraham.ac. $\mathrm{uk} /$ projects/trim galore/). Summary metrics were created across all samples using the MultiQC package (version 1.4, http://multiqc.info) ${ }^{71}$. FastQ files were merges using seqkit (version 0.8 .0$)^{72}$. Sequencing reads were aligned to the $\mathrm{C} 57 \mathrm{Bl} / 6 \mathrm{~J}$ reference genome (GRCm38, mm10) using BowTie2 with default parameters (version 2.3.4, http://bowtie-bio.sourceforge.net/bowtie2/index.shtml $)^{73}$. Duplicates were marked using Picard (version 2.9.0, http://broadinstitute.github.io/picard).

SV analysis was performed using Manta (version 0.29.6) ${ }^{74}$. SV were filtered using vcftools (version 0.1 .15$)^{75}$. In order to identify SNPs, the data were remapped to the $m m 10$ reference mouse genome using BWA (version 0.7.15-r1144- dirty) ${ }^{76}$. Reads were locally realigned and SNPs and short indels identified using GenomeAnalysisTK (GATK, version 3.7) ${ }^{77}$. Homozygous variants were called when $>90 \%$ of reads at the locus supported the variant call, whereas variants with at least $30 \%$ of reads supporting the variant calls were classified as heterozygous. Two rounds of filtering of variants were performed as follows. First, low-quality and biased variant calls were removed. Second, variants with: (i) simple repeats with a periodicity $<9 \mathrm{bp}$, (ii) homopolymer repeats $>8 \mathrm{bp}$, (iii) dinucleotide repeats $>14 \mathrm{bp}$, (iv) low mapping quality $(<40)$, (v) overlapping annotated repeats or segmental duplications, and (vi) $>3$ heterozygous variants fell within a $10 \mathrm{~kb}$ region were removed using vcftools (version 0.1.15) and bcftools (version 1.3.1) as was previously described ${ }^{78}$. The 129P2/OlaHsd mouse genome variation data were downloaded from Mouse Genomes Project ${ }^{79}$. The functional effect of SNPs was predicted using snpEff (version $4.3 \mathrm{t})^{80}$.

Methylated DNA immunoprecipitation and sequencing. MeDIP-seq ${ }^{81}$ was carried out using $3 \mu \mathrm{g}$ of sperm gDNA that was sonicated using a Diagenode Bioruptor UCD-200 to yield 200-700 bp fragments that were end-repaired and dAtailed. Illumina adaptors for paired-end sequencing were ligated using the NEB Next DNA Library Prep Master Mix for Illumina kit (New England Biolabs). After each step, the DNA was washed using Agencourt AMPure XP SPRI beads (Beckman Coulter). IPs were performed in triplicate using $500 \mathrm{ng}$ of DNA per sample, $1.25 \mu \mathrm{l}$ of mouse anti-human $5 \mathrm{mC}$ antibody $(0.1 \mathrm{mg} / 0.1 \mathrm{~mL}$, clone 33D3; Eurogentec Ltd., Cat No. BI-MECY, RRID:AB_2616058), and $10 \mu$ lof Dynabeads coupled with M-280 sheep anti-mouse IgG bead (Invitrogen). The three IPs were pooled and purified using MinElute PCR Purification columns (Qiagen). Libraries were amplified by PCR (12 cycles) using Phusion High-Fidelity PCR Master Mix and adaptor-specific iPCR tag primers (Supplementary Table 9), and purified using Agencourt AMPure XP SPRI beads. The efficiency of the IP was verified using qPCR to compare the enrichment for DNA regions of known methylation status (e.g., methylated in sperm: H19 and Peg3 ICR, Supplementary Fig. 5a) ${ }^{49}$ in the preamplification input and the IP fractions. MeDIP library DNA concentrations were estimated using the Kapa Library Quantification kit (Kapa Biosystems) and were further verified by running on an Agilent High Sensitivity DNA chip on an Agilent 2100 BioAnalyzer. Sequencing of MeDIP libraries was performed using $100 \mathrm{bp}$ paired-end reads on an Illumina HiSeq platform at the Babraham Institute Next Generation Sequencing Facility (Cambridge, UK).

Quality assessment of the sequencing reads was performed using FastQC (version 0.11.5, http://www.bioinformatics.babraham.ac.uk/projects/fastqc/). Adaptor trimming was performed using Trim Galore (version 0.6.4, http://www. bioinformatics.babraham.ac.uk/projects/trim galore/). Reads were mapped to the GRCm38 (mm10) reference genome using Bowtie2 (version 2.3.4, http://bowtiebio.sourceforge.net/bowtie2/index.shtml $)^{73}$. All programmes were run with default settings unless otherwise stated. Sample clustering was assessed using principle component analysis, using the 500 most variable windows with respect to read coverage (as a proxy for methylation) for $5 \mathrm{~kb}$ window across all samples. Further data quality checks and differential methylation analysis was performed using the MEDIPS package in $\mathrm{R}$ (version 1.40.0, using $\mathrm{R}$ version 3.4 .2 ) $^{33}$. The following key parameters were defined: BSgenome $=$ BSgenome.Mmusculus.UCSC.mm10, uniq $=1 \mathrm{e}-3$, extend $=300$, ws $=500$, shift $=0$. DMRs were defined as windows $(500 \mathrm{bp})$ in which there was at least 1.5-fold difference in methylation (reads per kilobase million mapped reads (RPKM)) between $\mathrm{C} 57 \mathrm{Bl} / 6 \mathrm{~J}$ and $\mathrm{Mtr}$ sperm methylation level with a $p$ value $<0.01$. Adjacent windows were merged using BEDTools (version 2.27.0) ${ }^{82}$. The background methylome was defined as all 500 bp windows across the genome at which the sum of the average RPKM per genotype group was $>1.0$. The genomic localisations of DMRs including association with coding/noncoding regions and $\mathrm{CpG}$ islands were determined using annotation downloaded from University of California, Santa Cruz (UCSC) ${ }^{83}$. The percentage of DMRs associated with repetitive regions of the genome was calculated using RepeatMasker software (version, http://www.repeatmasker.org).

Enrichment analysis of published ChIP-seq and ATAC-seq data. Mean enrichment of specific histone modifications and THSS, CTCF, H3.3 and PRM1 binding in the DMR regions were determined using published data sets including ChIP-seq data in CD1 spermatozoa collected from cauda epididymis ${ }^{42}, \mathrm{ESCs}^{35}$ and $\mathrm{TSCs}^{35}$, and ATAC-seq data in CD1 spermatozoa collected from cauda epididymis ${ }^{42}$ and B6D2F1 epiblast and extraembryonic ectoderm at E6.5 $5^{43}$. The source and accession numbers of processed ChIP-seq and ATAC-seq wig/bigwig files are shown in Supplementary Table 10 and accessible on GitHub (https://github.com/CTR-BFX Blake_Watson). To ensure that the analysis was consistent across public data sets, all wig files were converted to bigwig using UCSC tools "wigToBigWig -clip" (http:// hgdownload.soe.ucsc.edu/admin/exe/).

DMRs identified in sperm from all three Mtrr genotypes (MeDIP-seq analysis) were combined to generate a list of 893 DMRs. To prevent the inclusion of DMRs associated with genomic variation, the $20 \mathrm{Mb}$ region surrounding the Mtrr gene (Chr13:58060780-80060780) was identified as 129P2Ola/Hsd genomic sequence was masked. This resulted in 459 DMRs for subsequent analysis. The ChIP-seq and ATAC-seq files in sperm ${ }^{42}$ were originally aligned to mouse reference genome $\mathrm{mm} 9$. The files were converted to mouse reference genome mm10 to match the other published data sets in this analysis using LiftOver with the mm9ToMm10. over.chain (http://genome.ucsc.edu/cgi-bin/hgLiftOver). To identify the baseline enrichment profiles around the DMRs for specific histone modifications, THSS, CTCF, H3.3 or PRM1, a similar number of genomic regions were randomly selected using bedtools (v2.26.0 $)^{82}$ with the following command: "bedtools shuffle -i DMRs.bed -g Mus_GRCm38_chrall.fa.fai -chrom -seed 27442958 -noOverlapping -excl Mtrr_mask20Mb.bed". The DMR profiles were created using

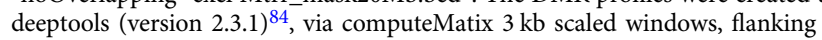
regions of $6 \mathrm{~kb}$, and a bin size of $200 \mathrm{bp}$, and plotted with plotProfile.

Enhancer analysis. To determine whether genetic variants or DMRs overlapped with known enhancer regions, FANTOM5 enhancer database ${ }^{85}$ for GRCm38 mouse genome (https://fantom.gsc.riken.jp/5/; F5.mm10.enhancers.bed.gz) was used. All.bed files were applied to UCSC Genome Browser ${ }^{83}$ to check for regionspecific features. The distance in base pairs between DMRs and genes or between enhancers and genes was calculated using the closest coordinates (the start/end of DMR or enhancer) to the transcriptional start site (TSS) minus the TSS and then plus 1 . To determine further interactions between enhancers and promoters, we analysed public Hi-C data sets ${ }^{35}$ for ESC and TSC (E-MTAB-6585; ESC_promoterother_interactions_GOTHiC.txt and

TSC_promoter-other_interactions_GOTHiC.txt) using WashU Epigenome Browser $^{86}$

Quantitative reverse transcription PCR (RT-qPCR). For RNA expression analysis, cDNA was synthesised using RevertAid $\mathrm{H}$ Minus reverse transcriptase (Thermo Scientific) and random hexamer primers (Thermo Scientific) using 1-2 $\mu \mathrm{g}$ of RNA in a $20-\mu \mathrm{l}$ reaction according to manufacturer's instructions. PCR amplification was conducted using MESA Green qPCR MasterMix Plus for SYBR Assay (Eurogentec Ltd.) on a DNA Engine Opticon2 thermocycler (BioRad). The following cycling conditions were used: $95^{\circ} \mathrm{C}$ for $10 \mathrm{~min}, 40$ cycles: $95^{\circ} \mathrm{C}$ for $30 \mathrm{sec}$ $60^{\circ} \mathrm{C}$ for $1 \mathrm{~min}$, followed by melt curve analysis. Transcript levels were normalised to Hprt and/or Gapdh RNA levels. Relative cDNA expression levels were analysed using the 2(-Delta Delta C(T)) method ${ }^{87}$. Experiments were conducted in technical triplicate with biological replicates indicated in the figure legends. Transcript levels in $\mathrm{C} 57 \mathrm{Bl} / 6 \mathrm{~J}$ tissue were normalised to 1 . For primer sequences and concentrations, refer to Supplementary Table 8 .

Bisulfite mutagenesis and pyrosequencing. Between $250 \mathrm{ng}$ and $2 \mu \mathrm{g}$ of gDNA extracted from each tissue sample was bisulfite treated with an Imprint DNA Modification Kit (Sigma). Control samples lacking DNA template were run to ensure that there was no contamination during the bisulfite conversion of DNA. To quantify DMR CpG methylation, pyrosequencing was performed. In all, $50 \mathrm{ng}$ of bisulfiteconverted DNA was used as a template for PCR, together with $0.2 \mu \mathrm{M}$ of each biotinylated primer and 0.25 units of HotStarTaq PlusDNA Polymerase (Qiagen). Refer to Supplementary Table 11 for primer sequences, which were designed using PyroMark Assay Design Software 2.0 (Qiagen). PCR was performed in triplicate 
using the following conditions: $95^{\circ} \mathrm{C}$ for $5 \mathrm{~min}, 40$ cycles of $94^{\circ} \mathrm{C}$ for $30 \mathrm{sec}, 56^{\circ} \mathrm{C}$ for $30 \mathrm{sec}, 72^{\circ} \mathrm{C}$ for $55 \mathrm{sec}$ and then $72^{\circ} \mathrm{C}$ for $5 \mathrm{~min}$. PCR products were purified using Strepdavidin Sepharose High Performance beads (GE healthcare). The beads bound to DNA were washed in $70 \%$ ethanol, $0.4 \mathrm{M} \mathrm{NaOH}$ and $10 \mathrm{mM}$ Tris-acetated $(\mathrm{pH}$ 7.6) and then hybridized to the sequencing primer in PyroMark annealing buffer (Qiagen) according to the manufacturer's instructions. Pyrosequencing was conducted using PyroMark Gold reagents kit (Qiagen) on a PyroMark MD pyrosequencer (Biotage). The mean CpG methylation was calculated using three to eight biological replicates and at least two technical replicates. Analysis of methylation status was performed using Pyro Q-CpG software (version 1.0.9, Biotage).

Mass spectrometry. Sperm gDNA was digested into individual nucleoside components using the DNA Degradase Plus kit (Zymo Research) according to the manufacturer's instructions. The heat inactivation step was omitted. In all, $100 \mathrm{ng}$ of degraded DNA per individual was sent to the Babraham Institute Mass Spectrometry Facility (Cambridge, UK), where global cytosine, $5 \mathrm{mC}$ and $5 \mathrm{hmC}$ was determined by liquid chromatography-tandem mass spectrometry (LC-MS/MS) as previously described ${ }^{88}$. The sperm of nine males were assessed per genotype and analysed in pools each containing three unique individuals. All pooled samples were analysed in triplicate. Global $5 \mathrm{mC}$ and $5 \mathrm{hmC}$ levels are reported as percentages relative to $\mathrm{C}$.

Western blotting. Embryos and placentas at E10.5 were homogenised in lysis buffer (20 mM Tris [pH 7.5], $150 \mathrm{mM} \mathrm{NaCl}, 1 \mathrm{mM}$ ethylenediaminetetraacetic acid (EDTA), $1 \mathrm{mM}$ ethylene glycol-bis( $\beta$-aminoethyl ether)-N,N, $\mathrm{N}^{\prime}, \mathrm{N}^{\prime}$-tetraacetic acid, $1 \%$ Triton X-100, $2.5 \mathrm{mM}$ sodium pyrophosphate, $1 \mathrm{mM} \beta$-glycerolphosphate, 1 $\mathrm{mM} \mathrm{Na} \mathrm{VO}_{4}$ and complete mini EDTA-free proteases inhibitor cocktail [Roche Diagnostics]) with Lysing Matrix D ceramic beads (MP Biomedical) using a MagNA Lyser (Roche Diagnostics) at $5500 \mathrm{rpm}$ for $20 \mathrm{sec}$. Samples were incubated on ice for $5 \mathrm{~min}$ and then homogenised again at $5500 \mathrm{rpm}$ for $20 \mathrm{sec}$. Homogenates were then incubated on ice for $20 \mathrm{~min}$ with brief intervening vortexing steps occurring every $5 \mathrm{~min}$. Samples were then centrifuged at $10,000 \times g$ for $5 \mathrm{~min}$. Supernatant from each sample was transferred to a new tube and centrifuged again at $10,000 \times g$ for $5 \mathrm{~min}$ to ensure that all residual tissue was removed. The protein concentration of tissue lysates was determined using bicinchoninic acid (SigmaAldrich). Proteins were denatured with gel loading buffer (50 mM Tris [pH 6.8], $100 \mathrm{mN}$ DTT, 2\% SDS, $10 \%$ glycerol and a trace amount of bromophenol blue) at $70{ }^{\circ} \mathrm{C}$ for $10 \mathrm{~min}$. Equivalent amounts of protein were resolved by $8-10 \%$ SDS-PAGE and blotted onto nitrocellulose $(0.2 \mu \mathrm{m}$, Amersham Protran) with a semi-dry blotter (GE Healthcare). The membrane was stained with Ponceau $S$ solution (Sigma-Aldrich) and the resulting scanned image was used as a loading control. After washing, the membrane was blotted with 5\% skimmed milk in Trisbuffered saline containing $0.1 \%$ Tween-20 (TBS-T) before incubation with 1:1000 dilution of monoclonal rabbit anti-human HIRA (clone D2A5E, Cell Signalling Technology, cat. No. 13307, RRID:AB_2798177) overnight at $4{ }^{\circ} \mathrm{C}$ or $1: 10,000$ dilution of monoclonal mouse anti-human $\beta$-actin (clone AC-74, Sigma-Aldrich, Cat. No. A2228, RRID:AB_476997) for $1 \mathrm{~h}$ at room temperature. Primary antibodies were diluted in TBS-T. The membrane was incubated with 1:10,000 dilution of donkey anti-rabbit IgG conjugated to horseradish peroxidase (HRP; GE Healthcare, cat. No. NA934, RRID:AB_772206) diluted in 2.5\% skimmed milk in TBS-T or 1:10,000 dilution of sheep anti-mouse IgG conjugated to HRP (GE Healthcare, cat. No. NA931V, RRID:AB_772210) diluted in TBS-T. The signal of resolved protein was visualized by Amersham enhanced chemiluminescence (ECL) Western Blotting Analysis System (GE Healthcare) using Amersham Hyperfilm ECL (GE Healthcare). A flat-bed scanner (HP Scanjet G4050) was used to scan films. Experiments were conducted in technical duplicates with the biological replicates indicated in the figure legends. Band intensities were determined with background subtraction using ImageJ (64-bit) software (version 1.48; NIH, USA). Full gel blots are available in the Source Data file.

Statistical analysis. Statistical analysis was performed using GraphPad Prism software (version 7). RT-qPCR data were analysed by independent two-tailed unpaired $t$ tests or ordinary one-way ANOVA with Dunnett's or Sidak's multiple comparison testing. SV and SNP data were analysed by independent two-tailed unpaired $t$ tests with Welch's correction. Bisulfite pyrosequencing data, SV chromosome frequency and DMR distribution at repetitive elements were analysed by two-way ANOVAs with Dunnett's, Sidak's or Tukey's multiple comparisons tests. A binomial test (Wilson/Brown) was used to compare the observed frequency of nucleosome occupancy at DMRs to expected values. Western blot data were analysed using a non-parametric Kruskal-Wallis test with Dunn's multiple comparisons test. $p<0.05$ was considered significant unless otherwise stated.

Reporting summary. Further information on research design is available in the Nature Research Reporting Summary linked to this article.

\section{Data availability}

All relevant data are available from the corresponding author upon reasonable request. WGS data have been deposited in ArrayExpress database at EMBL-EBI under accession number E-MTAB-8513 and MeDIP-Seq data accession number is E-MTAB-8533. The Hi-C data ${ }^{35}$ accession number is E-MTAB-6585. The source and accession numbers of processed ChIP-seq and ATAC-seq wig/bigwig files are listed in Supplementary Table 10. Source data are provided with this paper.

\section{Code availability}

The in-house scripts used for the analysis can be found freely accessible in the following online repository: https://github.com/CTR-BFX/Blake_Watson and https://doi.org/ 10.5281/zenodo.3832249).

Received: 12 May 2020; Accepted: 29 May 2021; Published online: 17 June 2021

\section{References}

1. Chen, Q. et al. Sperm tsRNAs contribute to intergenerational inheritance of an acquired metabolic disorder. Science 351, 397-400 (2016).

2. Gapp, K. et al. Implication of sperm RNAs in transgenerational inheritance of the effects of early trauma in mice. Nat. Neurosci. 17, 667-9 (2014).

3. Padmanabhan, N. et al. Mutation in folate metabolism causes epigenetic instability and transgenerational effects on development. Cell 155, 81-93 (2013)

4. Radford, E. J. et al. In utero effects. In utero undernourishment perturbs the adult sperm methylome and intergenerational metabolism. Science 345, 1255903 (2014).

5. Anway, M. D., Cupp, A. S., Uzumcu, M. \& Skinner, M. K. Epigenetic transgenerational actions of endocrine disruptors and male fertility. Science 308, 1466-9 (2005)

6. Blake, G. E. \& Watson, E. D. Unravelling the complex mechanisms of transgenerational epigenetic inheritance. Curr. Opin. Chem. Biol. 33, 101-7 (2016).

7. Heard, E. \& Martienssen, R. A. Transgenerational epigenetic inheritance: myths and mechanisms. Cell 157, 95-109 (2014).

8. Beck, D., Sadler-Riggleman, I. \& Skinner, M. K. Generational comparisons (F1 versus F3) of vinclozolin induced epigenetic transgenerational inheritance of sperm differential DNA methylation regions (epimutations) using MeDIPSeq. Environ. Epigenet. 3, dvx016 (2017)

9. Sharma, U. et al. Biogenesis and function of tRNA fragments during sperm maturation and fertilization in mammals. Science 351, 391-396 (2016).

10. Siklenka, K. et al. Disruption of histone methylation in developing sperm impairs offspring health transgenerationally. Science 350, aab2006 (2015).

11. Jimenez-Chillaron, J. C. et al. Intergenerational transmission of glucose intolerance and obesity by in utero undernutrition in mice. Diabetes $\mathbf{5 8}, \mathbf{4 6 0 - 8}$ (2009).

12. Elmore, C. L. et al. Metabolic derangement of methionine and folate metabolism in mice deficient in methionine synthase reductase. Mol. Genet. Metab. 91, 85-97 (2007).

13. Leclerc, D. et al. Cloning and mapping of a cDNA for methionine synthase reductase, a flavoprotein defective in patients with homocystinuria. Proc. Natl. Acad. Sci. USA 95, 3059-64 (1998).

14. Yamada, K., Gravel, R. A., Toraya, T. \& Matthews, R. G. Human methionine synthase reductase is a molecular chaperone for human methionine synthase. Proc. Natl. Acad. Sci. USA 103, 9476-81 (2006).

15. Field, M. S., Kamynina, E., Chon, J. \& Stover, P. J. Nuclear folate metabolism. Annu. Rev. Nutr. 38, 219-243 (2018).

16. Shane, B. \& Stokstad, E. L. Vitamin B12-folate interrelationships. Annu. Rev Nutr. 5, 115-41 (1985).

17. Ghandour, H., Lin, B. F., Choi, S. W., Mason, J. B. \& Selhub, J. Folate status and age affect the accumulation of L-isoaspartyl residues in rat liver proteins. J. Nutr. 132, 1357-60 (2002).

18. Jacob, R. A. et al. Moderate folate depletion increases plasma homocysteine and decreases lymphocyte DNA methylation in postmenopausal women. J. Nutr. 128, 1204-12 (1998).

19. Wainfan, E., Moller, M. L., Maschio, F. A. \& Balis, M. E. Ethionine-induced changes in rat liver transfer RNA methylation. Cancer Res. 35, 2830-5 (1975).

20. Wilson, A. et al. A common variant in methionine synthase reductase combined with low cobalamin (vitamin B12) increases risk for spina bifida. Mol. Genet. Metab. 67, 317-23 (1999).

21. Rosenblatt, D. S., Cooper, B. A., Schmutz, S. M., Zaleski, W. A. \& Casey, R. E Prenatal vitamin B12 therapy of a fetus with methylcobalamin deficiency (cobalamin E disease). Lancet 1, 1127-9 (1985).

22. Schuh, S. et al. Homocystinuria and megaloblastic anemia responsive to vitamin B12 therapy. An inborn error of metabolism due to a defect in cobalamin metabolism. N. Engl. J. Med. 310, 686-90 (1984).

23. Koury, M. J. \& Ponka, P. New insights into erythropoiesis: the roles of folate, vitamin B12, and iron. Annu Rev. Nutr. 24, 105-31 (2004). 
24. Padmanabhan, N. et al. Abnormal folate metabolism causes age-, sex- and parent-of-origin-specific haematological defects in mice. J. Physiol. 596, 4341-4360 (2018).

25. Nashun, B. et al. Continuous histone replacement by Hira is essential for normal transcriptional regulation and de novo DNA methylation during mouse iogenesis. Mol. Cell 60, 611-25 (2015).

26. Pchelintsev, N. A. et al. Placing the HIRA histone chaperone complex in the chromatin landscape. Cell Rep. 3, 1012-9 (2013).

27. Lin, C. J., Koh, F. M., Wong, P., Conti, M. \& Ramalho-Santos, M. Hiramediated H3.3 incorporation is required for DNA replication and ribosomal RNA transcription in the mouse zygote. Dev. Cell 30, 268-79 (2014).

28. Simon, M. M. et al. A comparative phenotypic and genomic analysis of C57BL/6J and C57BL/6N mouse strains. Genome Biol. 14, R82 (2013).

29. Kim, S. et al. PRMT5 protects genomic integrity during global DNA demethylation in primordial germ cells and preimplantation embryos. Mol. Cell 56, 564-79 (2014).

30. Deniz, O., Frost, J. M. \& Branco, M. R. Regulation of transposable elements by DNA modifications. Nat. Rev. Genet. 20, 417-431 (2019).

31. Blake, G. E. T., Hall, J., Petkovic, G. E. \& Watson, E. D. Analysis of spermatogenesis and fertility in adult mice with a hypomorphic mutation in the Mtrr gene. Reprod. Fertil. Dev. 31, 1730-1741 (2019).

32. Taiwo, O. et al. Methylome analysis using MeDIP-seq with low DNA concentrations. Nat. Protoc. 7, 617-36 (2012)

33. Lienhard, M., Grimm, C., Morkel, M., Herwig, R. \& Chavez, L. MEDIPS: genome-wide differential coverage analysis of sequencing data derived from DNA enrichment experiments. Bioinformatics 30, 284-6 (2014).

34. Orozco, L. D. et al. Epigenome-wide association of liver methylation patterns and complex metabolic traits in mice. Cell Metab. 21, 905-17 (2015).

35. Schoenfelder, S. et al. Divergent wiring of repressive and active chromatin interactions between mouse embryonic and trophoblast lineages. Nat. Commun. 9, 4189 (2018)

36. Watkins-Chow, D. E. \& Pavan, W. J. Genomic copy number and expression variation within the C57BL/6J inbred mouse strain. Genome Res 18, 60-6 (2008).

37. Casas, E. \& Vavouri, T. Sperm epigenomics: challenges and opportunities. Front. Genet. 5, 330 (2014).

38. Hisano, M. et al. Genome-wide chromatin analysis in mature mouse and human spermatozoa. Nat. Protoc. 8, 2449-70 (2013).

39. Brunner, A. M., Nanni, P. \& Mansuy, I. M. Epigenetic marking of sperm by post-translational modification of histones and protamines. Epigenetics Chromatin 7, 2 (2014)

40. Carone, B. R. et al. High-resolution mapping of chromatin packaging in mouse embryonic stem cells and sperm. Dev. Cell 30, 11-22 (2014).

41. Erkek, S. et al. Molecular determinants of nucleosome retention at CpG-rich sequences in mouse spermatozoa. Nat. Struct. Mol. Biol. 20, 868-75 (2013).

42. Jung, Y. H. et al. Chromatin states in mouse sperm correlate with embryonic and adult regulatory landscapes. Cell Rep. 18, 1366-1382 (2017).

43. Smith, Z. D. et al. Epigenetic restriction of extraembryonic lineages mirrors the somatic transition to cancer. Nature 549, 543-547 (2017).

44. Seah, M. K. Y. \& Messerschmidt, D. M. From germline to soma: epigenetic dynamics in the mouse preimplantation embryo. Curr. Top. Dev. Biol. 128, 203-235 (2018).

45. Tang, W. W., Kobayashi, T., Irie, N., Dietmann, S. \& Surani, M. A. Specification and epigenetic programming of the human germ line. Nat. Rev. Genet. 17, 585-600 (2016).

46. Hackett, J. A. et al. Germline DNA demethylation dynamics and imprint erasure through 5-hydroxymethylcytosine. Science 339, 448-52 (2013).

47. Kobayashi, H. et al. Contribution of intragenic DNA methylation in mouse gametic DNA methylomes to establish oocyte-specific heritable marks. PLoS Genet. 8, e1002440 (2012).

48. Smallwood, S. A. et al. Dynamic CpG island methylation landscape in oocytes and preimplantation embryos. Nat. Genet. 43, 811-4 (2011).

49. Lambrot, R. et al. Low paternal dietary folate alters the mouse sperm epigenome and is associated with negative pregnancy outcomes. Nat. Commun. 4, 2889 (2013)

50. Murphy, P. J. et al. NRF2 loss recapitulates heritable impacts of paternal cigarette smoke exposure. PLoS Genet. 16, e1008756 (2020).

51. Gardiner-Garden, M. \& Frommer, M. CpG islands in vertebrate genomes. J. Mol. Biol. 196, 261-82 (1987).

52. Roberts, C. et al. Targeted mutagenesis of the Hira gene results in gastrulation defects and patterning abnormalities of mesoendodermal derivatives prior to early embryonic lethality. Mol. Cell Biol. 22, 2318-28 (2002).

53. Batista, P. J. \& Chang, H. Y. Long noncoding RNAs: cellular address codes in development and disease. Cell 152, 1298-307 (2013).

54. Stover, P. J. One-carbon metabolism-genome interactions in folate-associated pathologies. J. Nutr. 139, 2402-5 (2009).

55. Blount, B. C. et al. Folate deficiency causes uracil misincorporation into human DNA and chromosome breakage: implications for cancer and neuronal damage. Proc. Natl. Acad. Sci. USA 94, 3290-5 (1997).
56. Bistulfi, G., Vandette, E., Matsui, S. \& Smiraglia, D. J. Mild folate deficiency induces genetic and epigenetic instability and phenotype changes in prostate cancer cells. BMC Biol. 8, 6 (2010).

57. Jablonka, E. Epigenetic inheritance and plasticity: the responsive germline. Prog. Biophys. Mol. Biol. 111, 99-107 (2013).

58. Miska, E. A. \& Ferguson-Smith, A. C. Transgenerational inheritance: models and mechanisms of non-DNA sequence-based inheritance. Science 354, 59-63 (2016).

59. Messerschmidt, D. M., Knowles, B. B. \& Solter, D. DNA methylation dynamics during epigenetic reprogramming in the germline and preimplantation embryos. Genes Dev. 28, 812-28 (2014).

60. Watson, E. D. \& Rakoczy, J. Fat eggs shape offspring health. Nat. Genet. 48, 478-9 (2016).

61. Zhang, Y. et al. Dnmt2 mediates intergenerational transmission of paternally acquired metabolic disorders through sperm small non-coding RNAs. Nat. Cell Biol. 20, 535-540 (2018).

62. Torne, J. et al. Two HIRA-dependent pathways mediate H3.3 de novo deposition and recycling during transcription. Nat. Struct. Mol. Biol. 27, 1057-1068 (2020)

63. Zhang, Y., Shi, J., Rassoulzadegan, M., Tuorto, F. \& Chen, Q. Sperm RNA code programmes the metabolic health of offspring. Nat. Rev. Endocrinol. 15, 489-498 (2019).

64. Holland, M. L. et al. Early-life nutrition modulates the epigenetic state of specific rDNA genetic variants in mice. Science 353, 495-8 (2016).

65. Gapp, K. et al. Alterations in sperm long RNA contribute to the epigenetic inheritance of the effects of postnatal trauma. Mol. Psychiatry 25, 2162-2174 (2018).

66. Branco, M. R. et al. Maternal DNA methylation regulates early trophoblast development. Dev. Cell 36, 152-63 (2016).

67. Park, S. J., Shirahige, K., Ohsugi, M. \& Nakai, K. DBTMEE: a database of transcriptome in mouse early embryos. Nucleic Acids Res. 43, D771-6 (2015)

68. Jiang, Z. et al. Zic3 is required in the extra-cardiac perinodal region of the lateral plate mesoderm for left-right patterning and heart development. Hum. Mol. Genet. 22, 879-89 (2013).

69. Tunster, S. J. Genetic sex determination of mice by simplex PCR. Biol. Sex. Differ. 8, 31 (2017).

70. Padmanabhan, N. et al. Multigenerational analysis of sex-specific phenotypic differences at midgestation caused by abnormal folate metabolism. Environ. Epigenet. 3, dvx014 (2017).

71. Ewels, P., Magnusson, M., Lundin, S. \& Kaller, M. MultiQC: summarize analysis results for multiple tools and samples in a single report. Bioinformatics 32, 3047-8 (2016).

72. Shen, W., Le, S., Li, Y. \& Hu, F. SeqKit: a cross-platform and ultrafast toolkit for FASTA/Q file manipulation. PLoS ONE 11, e0163962 (2016).

73. Langmead, B. \& Salzberg, S. L. Fast gapped-read alignment with Bowtie 2. Nat. Methods 9, 357-9 (2012).

74. Chen, X. et al. Manta: rapid detection of structural variants and indels for germline and cancer sequencing applications. Bioinformatics 32, 1220-2 (2016).

75. Danecek, P. et al. The variant call format and VCFtools. Bioinformatics 27, 2156-8 (2011).

76. Li, H. \& Durbin, R. Fast and accurate short read alignment with BurrowsWheeler transform. Bioinformatics 25, 1754-60 (2009).

77. McKenna, A. et al. The Genome Analysis Toolkit: a MapReduce framework for analyzing next-generation DNA sequencing data. Genome Res. 20, 1297-303 (2010).

78. Oey, H., Isbel, L., Hickey, P., Ebaid, B. \& Whitelaw, E. Genetic and epigenetic variation among inbred mouse littermates: identification of inter-individual differentially methylated regions. Epigenetics Chromatin 8, 54 (2015).

79. Keane, T. M. et al. Mouse genomic variation and its effect on phenotypes and gene regulation. Nature 477, 289-94 (2011).

80. Cingolani, P. et al. A program for annotating and predicting the effects of single nucleotide polymorphisms, SnpEff: SNPs in the genome of Drosophila melanogaster strain w1118; iso-2; iso-3. Fly. (Austin) 6, 80-92 (2012).

81. Senner, C. E., Krueger, F., Oxley, D., Andrews, S. \& Hemberger, M. DNA methylation profiles define stem cell identity and reveal a tight embryonicextraembryonic lineage boundary. Stem Cells 30, 2732-45 (2012).

82. Quinlan, A. R. \& Hall, I. M. BEDTools: a flexible suite of utilities for comparing genomic features. Bioinformatics 26, 841-2 (2010).

83. Meyer, L. R. et al. The UCSC Genome Browser database: extensions and updates 2013. Nucleic Acids Res. 41, D64-9 (2013).

84. Ramirez, F., Dundar, F., Diehl, S., Gruning, B. A. \& Manke, T. deepTools: a flexible platform for exploring deep-sequencing data. Nucleic Acids Res. 42, W187-91 (2014).

85. Andersson, R. et al. An atlas of active enhancers across human cell types and tissues. Nature 507, 455-461 (2014).

86. Li, D., Hsu, S., Purushotham, D., Sears, R. L. \& Wang, T. WashU epigenome browser update 2019. Nucleic Acids Res. 47, W158-W165 (2019). 
87. Livak, K. J. \& Schmittgen, T. D. Analysis of relative gene expression data using real-time quantitative PCR and the 2(-Delta Delta $\mathrm{C}(\mathrm{T})$ ) method. Methods 25, 402-8 (2001)

88. Ficz, G. et al. FGF signaling inhibition in ESCs drives rapid genome-wide demethylation to the epigenetic ground state of pluripotency. Cell Stem Cell 13, 351-9 (2013).

\section{Acknowledgements}

We thank Drs Nozomi Takahashi and Tessa Bertozzi for their technical support, Dr. Claire Senner for critical discussion, and Drs Jamie Hackett and Miguel Branco for .bed files. The MeDIP-seq and LC-MS/MS were performed at the Babraham Institute (UK). WGS was performed by BGI (Hong Kong). This work was funded by grants from Lister Institute for Preventative Medicine and the Centre for Trophoblast Research (CTR) to E. D.W and from the MRC MR/J001597 and Wellcome Trust WT095606 (to A.C.F.-S). The following support was provided: Wellcome Trust 4-year DTP in Developmental Mechanisms (to G.E.T.B.) and CTR funding (to G.E.T.B., H.W.Y., X.Z., R.S.H.).

\section{Author contributions}

E.D.W. conceived the project. G.E.T.B. collected sperm, performed DNA/RNA extractions, generated WGS and MeDIP libraries, and performed RT-qPCR and bisulfite pyrosequencing analyses. G.E.T.B. and E.D.W. dissected tissue and phenotyped conceptuses. G.E.T.B. and E.D.W. collected and analysed the data. X.Z., R.S.H. and G.E.T.B. designed and performed bioinformatics analyses. H.W.Y. performed the western blotting analysis. G.E.T.B., A.C.F.-S., G.J.B. and E.D.W. designed the experiments and interpreted the results. E.D.W. and G.E.T.B. wrote the manuscript. All authors read and revised the manuscript.

\section{Competing interests}

The authors declare no competing interests.

\section{Additional information}

Supplementary information The online version contains supplementary material available at https://doi.org/10.1038/s41467-021-24036-5.

Correspondence and requests for materials should be addressed to E.D.W.

Peer review information Nature Communications thanks Qi Chen, Jerome Jullien and the other, anonymous, reviewer(s) for their contribution to the peer review of this work. Peer reviewer reports are available.

Reprints and permission information is available at http://www.nature.com/reprints

Publisher's note Springer Nature remains neutral with regard to jurisdictional claims in published maps and institutional affiliations.

\begin{abstract}
(c) (i)
Open Access This article is licensed under a Creative Commons Attribution 4.0 International License, which permits use, sharing, adaptation, distribution and reproduction in any medium or format, as long as you give appropriate credit to the original author(s) and the source, provide a link to the Creative Commons license, and indicate if changes were made. The images or other third party material in this article are included in the article's Creative Commons license, unless indicated otherwise in a credit line to the material. If material is not included in the article's Creative Commons license and your intended use is not permitted by statutory regulation or exceeds the permitted use, you will need to obtain permission directly from the copyright holder. To view a copy of this license, visit http://creativecommons.org/ licenses/by/4.0/.
\end{abstract}

(C) The Author(s) 2021 